SOGANG-HEP 304/03

AEI-2003-018

hep-th/0302060

\title{
Superstrings and D-branes in A Plane Wave
}

\author{
Jongwook Kim ${ }^{a *}$, Bum-Hoon Lee ${ }^{a, b \dagger}$ and Hyun Seok Yang ${ }^{a \ddagger}$ \\ ${ }^{a}$ Department of Physics, Sogang University, Seoul 121-742, Korea \\ ${ }^{b}$ Max-Planck-Institut für Gravitationsphysik, \\ Albert-Einstein-Institut, Am Mühlenberg 1, D-14476 Golm, Germany
}

\begin{abstract}
We carefully analyze the supersymmetry algebra of closed strings and open strings in a type IIB plane wave background. We use eight component chiral spinors, $S O(8)$ MajoranaWeyl spinors, in light-cone gauge to provide a useful basis of string field theory calculation in the plane wave. We consider the two classes of D-branes, $D_{ \pm}$-branes, and give a worldsheet derivation of conserved supercurrents for all half BPS D-branes preserving 16 supersymmetries in the type IIB plane wave background. We exhaustively provide the supersymmetry algebra of the half BPS branes as well. We also point out that the supersymmetry algebra distinguishes the two $S O(4)$ directions with relative sign which is consistent with the $Z_{2}$ symmetry of the string action.
\end{abstract}

PACS numbers: 11.25.-w, 11.25.Uv

November 4, 2018

*jongwook@sogang.ac.kr

†bhl@ccs.sogang.ac.kr

†hsyang@hepth.sogang.ac.kr 


\section{Introduction}

The Penrose limit of the $A d S_{5} \times S^{5}$ background in type IIB supergravity corresponds to a plane wave solution [1],

$$
\begin{aligned}
& d s^{2}=-2 d x^{+} d x^{-}-\mu^{2} x_{I}^{2}\left(d x^{+}\right)^{2}+d x_{I}{ }^{2}, \\
& F_{+1234}=F_{+5678}=2 \mu .
\end{aligned}
$$

This implies a correspondence between type IIB string theory in the plane wave background (1.1) and $\mathcal{N}=4$ supersymmetric Yang-Mills theory with large R-charge, in a sense as a part of $A d S_{5} / C F T_{4}$ correspondence. Since the background (1.1) is one of the very few RamondRamond backgrounds on which string theory is exactly solvable $[2,3]$, one may have a genuine hope to explicitly check the conjectured AdS/CFT correspondence beyond the supergravity approximation on the string theory side. Indeed Berenstein, Maldacena and Nastase [4] succeeded in reproducing the string spectrum from perturbative super Yang-Mills theory, thereby putting the duality on a firm ground at the free theory level. Subsequent developments using the super Yang-Mills theory [5]-[12] and the light-cone string field theory [13]-[21] showed that the duality is still valid even after the interactions both on the super Yang-Mills theory side and on the string theory side are introduced.

D-branes can be described by boundary states of closed string state. The symmetries that the boundary state preserves are thus the combinations of the closed string symmetries that leave the boundary state invariant. Recently possible D-branes in the plane wave background (1.1) were identified and their supersymmetries were classified [22]-[30]. In particular, Skenderis and Taylor showed in $[28,29]$ that, although the kinematical supersymmetry descending from the closed string is totally broken on a $D_{+}$-brane $[27,30]$ (see section 2 for the definition of $D_{ \pm}$branes), a different kind of kinematical supersymmetry is nontrivially realized by incorporating the worldsheet symmetries. We will study this kinematical supersymmetry too.

Since the plane wave background (1.1) allows a light-cone gauge choice and the string theory in this background takes the simplest form in the light-cone gauge, the most straightforward method for constructing the superstring interactions is to use the light-cone Green-Schwarz formalism [2]. Since the original papers [31, 32, 33] on string field theory were using the 8-component spinors, $S O(8)$ Majorana-Weyl spinors, it may be desirable to provide the supersymmetry algebra in this basis as a useful reference for string field theory calculation in the plane wave, although detailed analysis had been done in $[2,3]$ for the closed string and in $[28,29]$ for the open string using 16-component spinors, $S O(9,1)$ Majorana-Weyl spinors. Thus we will exhaustively provide the supersymmetry algebras for both closed strings and open strings in the plane wave background using the 8-component spinors.

As is well known the spectrum of light-cone hamiltonian of plane wave superstring is dis-

crete. As analyzed in [34] recently, the super Yang-Mills theory in a four-dimensional plane 
wave background has also a discrete spectrum of light-cone energy operator, so expected to be most appropriate for establishing a precise correspondence with plane wave superstring and for studying the holographic issues of the duality. Since the plane wave super Yang-Mills theory is the low energy worldvolume action of probe D3-branes in the plane wave background (1.1) and is the ground-state sector of the open string field theory which also has a discrete energy spectrum, it is plausible that the plane wave super Yang-Mills theory including interactions can be captured by the open superstring field theory defined on the probe D3-branes [35]. Furthermore the open-closed string duality [25, 30], being, probably, an underlying principle of AdS/CFT duality, implies that the open string field theory possibly reproduces the corresponding results of closed string field theory. For this purpose it will be useful to explicitly construct the supersymmetry algebra for the open string in the plane wave background (1.1) using 8-component spinors mostly used in the light-cone string field theory [31, 33].

This paper is organized as follows. In Sec. 2, we discuss the canonical quantization of superstrings in the plane wave background and present the mode expansions of open strings compatible with boundary conditions. In Sec. 3, we first analyze the supersymmetry algebra of closed string to provide its explicit expressions in terms of 8-component spinors. We find that the supersymmetry algebra distinguishes the two $S O(4)$ directions with opposite sign consistent with the $Z_{2}$ symmetry of the string action [18]. We derive conserved supercurrents for all half BPS D-branes preserving 16 supersymmetries in the type IIB plane wave background and exhaustively provide the mode expansion of symmetry generators and supersymmetry algebra for half BPS D-branes, including D-branes with worldvolume flux which were not studied in $[28,29]$, using 8-component spinors. In Sec. 4, we briefly review our results and address some other issues. In Appendix A, we explain our notations and definitions and give useful formula used in this paper. In Appendix B, we provide some technical details on (anti-)commutation relations for $D_{+}$-branes. In Appendix $\mathrm{C}$, we present how the kinematical supersymmetry of $D_{+}$-brane can be derived from the open string mode expansion.

\section{Canonical Quantization of Superstrings in Plane Wave}

The Green-Schwarz light-cone action in the plane wave background (1.1) describes eight free massive bosons and fermions. In the light-cone gauge, $X^{+}=\tau$, the action is given by

$$
S=\frac{1}{2 \pi \alpha^{\prime} p^{+}} \int d \tau \int_{0}^{2 \pi \alpha^{\prime}\left|p^{+}\right|} d \sigma\left[\frac{1}{2} \partial_{+} X_{I} \partial_{-} X_{I}-\frac{1}{2} \mu^{2} X_{I}^{2}-i \bar{S}\left(\rho^{A} \partial_{A}-\mu \Pi\right) S\right]
$$

where $\partial_{ \pm}=\partial_{\tau} \pm \partial_{\sigma}$. Our notations and conventions are summarized in Appendix A. In this paper we will take $\alpha=\alpha^{\prime} p^{+}$for closed string and $\alpha=2 \alpha^{\prime} p^{+}$for open string. We take the spinor $S$ as eight two-component Majorana spinors on the worldsheet $\Sigma$ that transform as positive 
chirality spinors $\mathbf{8}_{s}$ under $S O(8)^{1}$ :

$$
S^{a}=\left(\begin{array}{c}
S^{1 a} \\
S^{2 a}
\end{array}\right), \quad \bar{S}^{a}=S^{a T} \rho^{\tau},
$$

where

$$
\rho^{\tau}=\left(\begin{array}{cc}
0 & -1 \\
1 & 0
\end{array}\right), \quad \rho^{\sigma}=\left(\begin{array}{ll}
0 & 1 \\
1 & 0
\end{array}\right) .
$$

The presence of $\Pi$ in the fermionic action breaks the symmetry from $S O(8)$ to $S O(4) \times S O(4)^{\prime}$. The equations of motion following from the action (2.1) take the form

$$
\begin{aligned}
& \partial_{+} \partial_{-} X^{I}+\mu^{2} X^{I}=0, \\
& \partial_{+} S^{1}-\mu \Pi S^{2}=0, \quad \partial_{-} S^{2}+\mu \Pi S^{1}=0 .
\end{aligned}
$$

\subsection{Closed string}

First we analyze the closed string case. Detailed analysis using 16-component spinors had been done in $[2,3]$ for the closed string and in $[28,29]$ for open strings. We will instead use 8-component spinors as in the original papers $[31,32,33]$ on string field theory. This will be a useful basis for light-cone string field theory and for open string analysis.

The general solutions to Eqs. (2.4) and (2.5) for the closed string are found to be ${ }^{2}$

$$
\begin{aligned}
& X^{I}(\sigma, \tau)=\cos \mu \tau x_{0}^{I}+\sin \mu \tau \frac{p_{0}^{I}}{\mu}+i \sum_{n \neq 0} \frac{1}{\omega_{n}}\left(\varphi_{n}^{1}(\sigma, \tau) \alpha_{n}^{1 I}+\varphi_{n}^{2}(\sigma, \tau) \alpha_{n}^{2 I}\right), \\
& S^{1}(\sigma, \tau)=\cos \mu \tau S_{0}^{1}+\sin \mu \tau \Pi S_{0}^{2}+\sum_{n \neq 0} c_{n}\left(\varphi_{n}^{1}(\sigma, \tau) S_{n}^{1}+i \rho_{n} \varphi_{n}^{2}(\sigma, \tau) \Pi S_{n}^{2}\right), \\
& S^{2}(\sigma, \tau)=\cos \mu \tau S_{0}^{2}-\sin \mu \tau \Pi S_{0}^{1}+\sum_{n \neq 0} c_{n}\left(\varphi_{n}^{2}(\sigma, \tau) S_{n}^{2}-i \rho_{n} \varphi_{n}^{1}(\sigma, \tau) \Pi S_{n}^{1}\right),
\end{aligned}
$$

where the basis functions $\varphi_{n}^{1,2}(\sigma, \tau)$ are defined by

$$
\varphi_{n}^{1}(\sigma, \tau)=e^{-i\left(\omega_{n} \tau-\frac{n}{|\alpha|} \sigma\right)}, \quad \varphi_{n}^{2}(\sigma, \tau)=e^{-i\left(\omega_{n} \tau+\frac{n}{|\alpha|} \sigma\right)}
$$

and

$$
\omega_{n}=\operatorname{sgn}(n) \sqrt{\mu^{2}+n^{2} / \alpha^{2}}, \quad \rho_{n}=\frac{\omega_{n}-n /|\alpha|}{\mu}, \quad c_{n}=\frac{1}{\sqrt{1+\rho_{n}^{2}}} .
$$

\footnotetext{
${ }^{1}$ The spinors $S^{A}$ are two $S O(8)$ Majorana-Weyl spinors satisfying the light-cone gauge. These spinors can be obtained by the original $S O(9,1)$ Majorana-Weyl spinors $\theta^{A}$ satisfying $\bar{\gamma}^{+} \theta^{A}=0$, fixing the $\kappa$-symmetry. As in (A.12), the fermionic light-cone gauge can be solved by taking $S^{A}=-\frac{1}{2} \gamma^{+} \bar{\gamma}^{-} \theta^{A}$.

${ }^{2}$ In our following analysis the mode expansion of string fields will be performed for real fields. Thus the reality of the field requires that $\xi_{n}^{\dagger}=\xi_{-n}$ for any bosonic or fermionic n-th mode $\xi_{n}$.
} 
For canonical quantization let us introduce the canonical momenta

$$
P^{I}(\sigma, \tau)=\frac{1}{2 \pi|\alpha|} \dot{X}^{I}(\sigma, \tau), \quad P^{A a}(\sigma, \tau)=\frac{i}{2 \pi|\alpha|} S^{A a}(\sigma, \tau),
$$

where $\dot{X}^{I}=\frac{\partial x^{I}}{\partial \tau}$. After removing the second class constraint in the fermionic part by introducing the Dirac bracket, we get the following equal-time quantum (anti-)commutation relations

$$
\begin{aligned}
& {\left[X^{I}(\sigma, \tau), \dot{X}^{J}\left(\sigma^{\prime}, \tau\right)\right]=i 2 \pi|\alpha| \delta^{I J} \delta\left(\sigma-\sigma^{\prime}\right),} \\
& \left\{S^{A a}(\sigma, \tau), S^{B b}\left(\sigma^{\prime}, \tau\right)\right\}=\pi|\alpha| \delta^{a b} \delta^{A B} \delta\left(\sigma-\sigma^{\prime}\right) .
\end{aligned}
$$

The above quantization gives the (anti-)commutation relations for the modes in (2.6) and (2.7):

$$
\begin{aligned}
& {\left[x_{0}^{I}, p_{0}^{J}\right]=i \delta^{I J}, \quad\left[\alpha_{n}^{I A}, \alpha_{m}^{J B}\right]=\frac{1}{2} \omega_{n} \delta_{m+n, 0} \delta^{I J} \delta^{A B},} \\
& \left\{S_{n}^{A a}, S_{m}^{B b}\right\}=\frac{1}{2} \delta_{n+m, 0} \delta^{a b} \delta^{A B} .
\end{aligned}
$$

\subsection{Open string}

Now we will discuss open strings living on a D-brane in the plane wave background (1.1). Here we consider only static D-branes for simplicity. To describe a Dp-brane, we impose the Neumann boundary conditions on $(p-1)$ coordinates and Dirichlet boundary conditions on the remaining transverse coordinates:

$$
\begin{aligned}
& \left.\partial_{\sigma} X^{r}\right|_{\partial \Sigma}=0 \\
& \left.\partial_{\tau} X^{r^{\prime}}\right|_{\partial \Sigma}=0 .
\end{aligned}
$$

For the fermionic coordinates, the appropriate boundary condition [36] is

$$
\left.S^{1}\right|_{\partial \Sigma}=\left.\Omega S^{2}\right|_{\partial \Sigma}
$$

It turns out $[28,29,30]$ that in the plane wave background there are two classes of maximally supersymmetric Dp-branes, depending on the choice of $\Omega$ :

$$
D_{-}: \Pi \Omega \Pi \Omega=-1, \quad D_{+}: \Pi \Omega \Pi \Omega=1 .
$$

The boundary conditions, $D_{ \pm}$, in (2.18) together with the fermionic equations of motion, $(2.5)$, imply [28] that

$$
\begin{aligned}
& D_{-}:\left.\partial_{\sigma} S^{1}\right|_{\partial \Sigma}=-\left.\Omega \partial_{\sigma} S^{2}\right|_{\partial \Sigma}, \\
& D_{+}:\left.\partial_{\sigma} S^{1}\right|_{\partial \Sigma}=\left.\left(-\Omega \partial_{\sigma} S^{2}+2 \mu \Pi S^{2}\right)\right|_{\partial \Sigma} .
\end{aligned}
$$


The boundary condition for $X^{-}$is determined by the Virasoro constraint

$$
\partial_{\sigma} X^{-}=\partial_{\tau} X^{I} \partial_{\sigma} X^{I}-\frac{i}{2}\left(S^{1} \partial_{+} S^{1}-S^{2} \partial_{-} S^{2}\right)
$$

from which one can see that the $X^{-}$coordinate must satisfy the Neumann boundary condition for both classes in (2.18).

Here we list the allowed choices for $\Omega$ consistent with each constraint in Eq. (2.18). For $D_{-}$-branes, there are the following possibilities [23, 24, 28, 30] (for the notation used below, see Appendix A):

$$
\begin{aligned}
& \text { D3: }(m, n)=(2,0),(0,2), \\
& \text { D5: }(m, n)=(3,1),(1,3), \\
& \text { D7: }(m, n)=(4,2),(2,4) .
\end{aligned}
$$

For $D_{+}$-branes, there are the following possibilities $[24,28,30]$ :

$$
\begin{aligned}
& \text { D1:(m,n) }=(0,0), \\
& \text { D3:(m,n) }=(1,1), \\
& \text { D5:(m,n) }=(4,0),(2,2),(0,4), \\
& \text { D7:(m,n) }=(3,3), \\
& \text { D9:(m,n) }=(4,4) .
\end{aligned}
$$

The quantization of open strings on $D_{-}$and $D_{+}$branes is defined by the following equal-time quantum (anti-)commutation relations

$$
\begin{aligned}
& {\left[X^{I}(\sigma, \tau), \dot{X}^{J}\left(\sigma^{\prime}, \tau\right)\right]=i \pi|\alpha| \delta^{I J} \delta\left(\sigma-\sigma^{\prime}\right)} \\
& \left\{S^{A a}(\sigma, \tau), S^{B b}\left(\sigma^{\prime}, \tau\right)\right\}=\frac{1}{2} \pi|\alpha| \delta^{a b} \delta^{A B} \delta\left(\sigma-\sigma^{\prime}\right) .
\end{aligned}
$$

For an open string, we have the boundary conditions on the fields, Eqs. (2.15)-(2.17). These boundary conditions will be incorporated in the mode expansion of the fields.

\subsubsection{D_-brane}

We first discuss the quantization of open strings on $D_{-}$-brane. The mode expansion of the bosonic coordinates satisfying the boundary condition and the equation of motion is given by

$$
\begin{aligned}
& X^{r}(\sigma, \tau)=\cos \mu \tau x_{0}^{r}+\sin \mu \tau \frac{p_{0}^{r}}{\mu}+i \sum_{n \neq 0} \frac{1}{\omega_{n}} \alpha_{n}^{r} e^{-i \omega_{n} \tau} \cos \frac{n \sigma}{|\alpha|} \\
& X^{r^{\prime}}(\sigma, \tau)=x_{0}^{r^{\prime}}(\sigma)+\sum_{n \neq 0} \frac{1}{\omega_{n}} \alpha_{n}^{r^{\prime}} e^{-i \omega_{n} \tau} \sin \frac{n \sigma}{|\alpha|}
\end{aligned}
$$


where the zero mode part, $x_{0}^{r^{\prime}}(\sigma)$, represents a D-brane position located either at the origin or away from the origin and is given by

$$
x_{0}^{r^{\prime}}(\sigma)=\frac{x_{0}^{r^{\prime}}}{1+e^{\mu|\alpha| \pi}}\left(e^{\mu \sigma}+e^{\mu(|\alpha| \pi-\sigma)}\right) .
$$

Similarly the mode expansion of the fermion is found to be

$$
\begin{aligned}
& S^{1}(\sigma, \tau)=\cos \mu \tau S_{0}-\sin \mu \tau \Omega \Pi S_{0}+\sum_{n \neq 0} c_{n}\left(\varphi_{n}^{1}(\sigma, \tau) \Omega S_{n}+i \rho_{n} \varphi_{n}^{2}(\sigma, \tau) \Pi S_{n}\right), \\
& S^{2}(\sigma, \tau)=\cos \mu \tau \Omega^{T} S_{0}-\sin \mu \tau \Pi S_{0}+\sum_{n \neq 0} c_{n}\left(\varphi_{n}^{2}(\sigma, \tau) S_{n}-i \rho_{n} \varphi_{n}^{1}(\sigma, \tau) \Pi \Omega S_{n}\right)
\end{aligned}
$$

One can check that the mode expansion (2.28) satisfies the boundary condition (2.17) and (2.19) as well as the equation of motion (2.5).

The commutation relations for the modes in Eqs. (2.26) and (2.28) are determined by the field quantization in (2.24) and (2.25) to be respectively

$$
\begin{aligned}
& {\left[x_{0}^{r}, p_{0}^{s}\right]=i \delta^{r s}, \quad\left[\alpha_{n}^{I}, \alpha_{m}^{J}\right]=\omega_{n} \delta_{m+n, 0} \delta^{I J},} \\
& \left\{S_{n}^{a}, S_{m}^{b}\right\}=\frac{1}{4} \delta_{n+m, 0} \delta^{a b}, \quad(n, m \in \mathbf{Z}) .
\end{aligned}
$$

\subsection{2 $D_{+}$-brane}

The mode expansion of the bosons is exactly the same as the $D_{-}$-branes, Eq. (2.26), and for the fermions we find that

$$
\begin{aligned}
& S^{1}(\sigma, \tau)=\cosh \mu \sigma S_{0}+\sinh \mu \sigma \Omega \Pi S_{0}+\sum_{n \neq 0} c_{n}\left(\varphi_{n}^{1}(\sigma, \tau) \widetilde{S}_{n}+i \rho_{n} \varphi_{n}^{2}(\sigma, \tau) \Pi S_{n}\right), \\
& S^{2}(\sigma, \tau)=\cosh \mu \sigma \Omega^{T} S_{0}+\sinh \mu \sigma \Pi S_{0}+\sum_{n \neq 0} c_{n}\left(\varphi_{n}^{2}(\sigma, \tau) S_{n}-i \rho_{n} \varphi_{n}^{1}(\sigma, \tau) \Pi \widetilde{S}_{n}\right),
\end{aligned}
$$

where

$$
\widetilde{S}_{n}=\frac{1}{\omega_{n}}\left(\frac{n}{|\alpha|} \Omega-i \mu \Pi\right) S_{n}
$$

One can check that the mode expansion (2.31) satisfies the boundary conditions, Eqs. (2.17) and (2.20), as well as the equation of motion, Eq. (2.5). For the $D_{+}$-branes, the zero modes of the fermions depend on the worldsheet space coordinate $\sigma$, and so there is no direct relation with the zero modes of the closed string [28].

The anti-commutation relation of the modes for $D_{+}$-branes is not trivial and some technical details are given in Appendix B:

$$
\begin{aligned}
& \left\{S_{0}^{a}, S_{0}^{b}\right\}=\frac{\pi \mu|\alpha|}{4 \sinh \pi \mu|\alpha|}\left(\delta^{a b} \cosh \pi \mu|\alpha|-(\Omega \Pi)^{a b} \sinh \pi \mu|\alpha|\right), \\
& \left\{S_{n}^{a}, S_{m}^{b}\right\}=\frac{1}{4} \delta_{n+m, 0} \delta^{a b}, \quad(n, m \neq 0) .
\end{aligned}
$$


Note that the result (2.33) has the correct flat space limit, $\mu \rightarrow 0$, including coefficient. Using the relation $(2.32)$ for the nonzero mode $\widetilde{S}_{n}$, the following anti-commutation relations can be derived from Eq. (2.34):

$$
\begin{aligned}
& \left\{\widetilde{S}_{n}^{a}, \widetilde{S}_{m}^{b}\right\}=\frac{1}{4} \delta_{n+m, 0} \delta^{a b}, \quad(n, m \neq 0), \\
& \left\{\widetilde{S}_{n}^{a}, S_{m}^{b}\right\}=\frac{1}{4 \omega_{n}}\left(\frac{n}{|\alpha|} \Omega_{a b}-i \mu \Pi_{a b}\right) \delta_{n+m, 0} .
\end{aligned}
$$

As shown in next section, for the D5-branes of type $(4,0)$ or $(0,4)$ to be supersymmetric, a nontrivial flux of gauge field is necessarily turned on in the worldvolume. The inclusion of the gauge field corresponds to the addition of the following boundary term

$$
S_{B}=\frac{1}{2 \pi \alpha^{\prime} p^{+}} \int d \tau \int_{0}^{2 \pi \alpha^{\prime}\left|p^{+}\right|} d \sigma F^{-r} \partial_{\sigma} X^{r}=\frac{\mu}{4 \pi \alpha^{\prime} p^{+}} \int_{\partial \Sigma} d \tau X^{r} X^{r}
$$

where the Born-Infeld flux $F^{-r}$ is given by

$$
F^{-r}=\mu X^{r}
$$

This affects the Neumann boundary condition and the appropriate boundary condition turns out to be [30]

$$
\left.\partial_{\sigma} X^{r}\right|_{\partial \Sigma}=\left.\mu X^{r}\right|_{\partial \Sigma}
$$

The boundary coupling in Eq. (2.37) is indeed generated by the superpotential in the $\mathcal{N}=(2,2)$ worldsheet supersymmetric theory [27]. The mode expansion of the Neumann coordinates $X^{r}(\sigma, \tau)$ is then given by

$$
\begin{aligned}
X^{r}(\sigma, \tau)= & \sqrt{\frac{2 \pi \mu|\alpha|}{e^{2 \pi \mu|\alpha|}-1}}\left(x_{0}^{r}+p_{0}^{r} \tau\right) e^{\mu \sigma}+i \sum_{n \neq 0} \frac{n}{\omega_{n}(n-i \mu|\alpha|)} \alpha_{n}^{r} e^{-i \omega_{n} \tau} \cos \frac{n \sigma}{|\alpha|} \\
& +i \sum_{n \neq 0} \frac{\mu|\alpha|}{\omega_{n}(n-i \mu|\alpha|)} \alpha_{n}^{r} e^{-i \omega_{n} \tau} \sin \frac{n \sigma}{|\alpha|} .
\end{aligned}
$$

The commutation relation between the modes is given by Eq. (2.29) as usual. See Appendix B for the derivation.

\section{Supersymmetry Algebra in Plane Wave}

We now study the basic symmetry algebra of the light-cone superstring in the plane wave described by the action (2.1). The thirty bosonic symmetries are generated by the ten translation generators, $P^{-}=H, P^{+}, P^{I}$, the eight boost generators, $J^{+I}$, the six $S O(4)$ rotation generators, $J^{i j}$, and the six $S O(4)^{\prime}$ rotation generators, $J^{i^{\prime} j^{\prime}}$. There are also 32 supersymmetries. In the light-cone gauge the 32 components of the supersymmetries decompose into 'dynamical' 
and 'kinematical' components. The dynamical supercharges, $Q_{\dot{a}}^{-A}$, commutes with the lightcone hamiltonian, but the kinematical supercharges, $Q_{a}^{+A}$, for closed strings and $D_{-}$-branes do not due to a fermionic mass term. However we will show that all supersymmetries commute with the hamiltonian for $D_{+}$-branes since the fermionic mass term identically vanishes.

Using the Nöther method, the symmetry generating charges can be obtained from conserved currents. For the superstring in the plane wave background described by the action (2.1), the super-Nöther charges were obtained by Metsaev [2]:

$$
\begin{aligned}
& P^{+}=p^{+}, \quad P^{I}=\frac{1}{2 \pi \alpha^{\prime} p^{+}} \int_{0}^{2 \pi \alpha^{\prime}\left|p^{+}\right|} d \sigma\left(\dot{X}^{I} \cos \mu \tau+\mu X^{I} \sin \mu \tau\right), \\
& 2 p^{+} H=\frac{1}{2 \pi \alpha^{\prime} p^{+}} \int_{0}^{2 \pi \alpha^{\prime}\left|p^{+}\right|} d \sigma\left[\frac{1}{2}\left(\dot{X}_{I}^{2}+X_{I}^{\prime 2}+\mu^{2} X_{I}^{2}\right)+i S^{A} \dot{S}^{A}\right] \\
& J^{+I}=\frac{1}{2 \pi \alpha^{\prime}} \int_{0}^{2 \pi \alpha^{\prime}\left|p^{+}\right|} d \sigma\left(\frac{\dot{X}^{I}}{\mu} \sin \mu \tau-X^{I} \cos \mu \tau\right) \\
& J^{i j}=\frac{1}{2 \pi \alpha^{\prime} p^{+}} \int_{0}^{2 \pi \alpha^{\prime}\left|p^{+}\right|} d \sigma\left[\left(X^{i} \dot{X}^{j}-X^{j} \dot{X}^{i}\right)-\frac{i}{2} S^{A} \gamma^{i j} S^{A}\right] \\
& J^{i^{\prime} j^{\prime}}=\frac{1}{2 \pi \alpha^{\prime} p^{+}} \int_{0}^{2 \pi \alpha^{\prime}\left|p^{+}\right|} d \sigma\left[\left(X^{i^{\prime}} \dot{X}^{j^{\prime}}-X^{j^{\prime}} \dot{X}^{i^{\prime}}\right)-\frac{i}{2} S^{A} \gamma^{\left.i^{\prime} j^{\prime} S^{A}\right]}\right. \\
& Q^{+}=\frac{\sqrt{2 p^{+}}}{2 \pi \alpha^{\prime} p^{+}} \int_{0}^{2 \pi \alpha^{\prime}\left|p^{+}\right|} d \sigma e^{i \mu \tau \Pi}\left(S^{1}+i S^{2}\right) \\
& \bar{Q}^{+}=\frac{\sqrt{2 p^{+}}}{2 \pi \alpha^{\prime} p^{+}} \int_{0}^{2 \pi \alpha^{\prime}\left|p^{+}\right|} d \sigma e^{-i \mu \tau \Pi}\left(S^{1}-i S^{2}\right), \\
& \sqrt{2 p^{+}} Q^{-1}=\frac{1}{2 \pi \alpha^{\prime} p^{+}} \int_{0}^{2 \pi \alpha^{\prime}\left|p^{+}\right|} d \sigma\left(\partial_{-} X^{I} \gamma^{I} S^{1}-\mu X_{I} \gamma^{I} \Pi S^{2}\right) \\
& \sqrt{2 p^{+}} Q^{-2}=\frac{1}{2 \pi \alpha^{\prime} p^{+}} \int_{0}^{2 \pi \alpha^{\prime}\left|p^{+}\right|} d \sigma\left(\partial_{+} X^{I} \gamma^{I} S^{2}+\mu X_{I} \gamma^{I} \Pi S^{1}\right)
\end{aligned}
$$

where $X_{I}^{\prime}=\frac{\partial X_{I}}{\partial \sigma}$.

In the light-cone formalism, the generators of the basic superalgebra can be split into the kinematical generators

$$
P^{+}, \quad P^{I}, \quad J^{+I}, \quad J^{i j}, \quad J^{i^{\prime} j^{\prime}}, Q_{a}^{+}, \bar{Q}_{a}^{+},
$$

and the dynamical generators

$$
H, Q_{\dot{a}}^{-1}, Q_{\dot{a}}^{-2} .
$$

Note that the kinematical supersymmetry generators have $S O(8)$ positive chirality while the dynamical supersymmetry generators have $S O(8)$ negative chirality. The kinematical generators $P^{+}, P^{I}, J^{+I}, Q_{a}^{+}, \bar{Q}_{a}^{+}$depend only on the zero modes since they are effectively linear in fields. Now we will study the supersymmetry algebra of the symmetry generating charges, (3.1)-(3.9), for the closed and the open strings, using the mode expansion given in the previous section. 


\subsection{Closed string}

Using the mode expansion in Eqs. (2.6) and (2.7), we get

$$
\begin{aligned}
& P^{+}=p^{+}, \quad P^{I}=p_{0}^{I}, \quad J^{+I}=-x_{0}^{I} p^{+}, \\
& Q^{+}=\sqrt{2 p^{+}}\left(S_{0}^{1}+i S_{0}^{2}\right)=\sqrt{2 p^{+}} S_{0}^{+}, \quad \bar{Q}^{+}=\sqrt{2 p^{+}}\left(S_{0}^{1}-i S_{0}^{2}\right)=\sqrt{2 p^{+}} S_{0}^{-} .
\end{aligned}
$$

The rotation generators $J^{I J}=\left(J^{i j} \in S O(4), J^{i^{\prime} j^{\prime}} \in S O(4)^{\prime}\right)$ are given by

$$
J^{I J}=x_{0}^{I} p_{0}^{J}-x_{0}^{J} p_{0}^{I}-\frac{i}{2} S_{0}^{A} \gamma^{I J} S_{0}^{A}-i \sum_{n \neq 0}\left\{\frac{1}{\omega_{n}}\left(\alpha_{-n}^{A I} \alpha_{n}^{A J}-\alpha_{-n}^{A J} \alpha_{n}^{A I}\right)+\frac{1}{2} S_{-n}^{A} \gamma^{I J} S_{n}^{A}\right\} .
$$

For the dynamical generators, we have

$$
\begin{aligned}
& 2 p^{+} H=\frac{1}{2}\left(p_{0 I}^{2}+\mu^{2} x_{0 I}^{2}\right)+2 i \mu S_{0}^{1} \Pi S_{0}^{2}+\sum_{n \neq 0}\left\{\alpha_{-n}^{A I} \alpha_{n}^{A I}+\omega_{n} S_{-n}^{A} S_{n}^{A}\right\} \\
& \sqrt{2 p^{+}} Q^{-1}=p_{0 I} \gamma^{I} S_{0}^{1}-\mu x_{0 I} \gamma^{I} \Pi S_{0}^{2}+2 \sum_{n \neq 0}\left\{c_{n} \alpha_{-n}^{1 I} \gamma^{I} S_{n}^{1}+\frac{i \mu}{2 c_{n} \omega_{n}} \alpha_{-n}^{2 I} \gamma^{I} \Pi S_{n}^{2}\right\} \\
& \sqrt{2 p^{+}} Q^{-2}=p_{0 I} \gamma^{I} S_{0}^{2}+\mu x_{0 I} \gamma^{I} \Pi S_{0}^{1}+2 \sum_{n \neq 0}\left\{c_{n} \alpha_{-n}^{2 I} \gamma^{I} S_{n}^{2}-\frac{i \mu}{2 c_{n} \omega_{n}} \alpha_{-n}^{1 I} \gamma^{I} \Pi S_{n}^{1}\right\}
\end{aligned}
$$

Now, from Eqs. (3.12)-(3.17), it is straightforward to derive the supersymmetry algebra of the closed string in the plane wave background [3] using the (anti-)commutation relations, (2.13) and (2.14). (Or one can directly calculate it from the super-Nöther charges, Eqs. (3.1)(3.9), using the (anti-)commutation relations, (2.11) and (2.12).) Here we will present only the non-vanishing (anti-)commutation relations involved with odd generators, $Q^{ \pm}$and $\bar{Q}^{ \pm}=\left(Q^{ \pm}\right)^{\dagger}$, where $Q_{\dot{a}}^{-}=Q_{\dot{a}}^{-1}+i Q_{\dot{a}}^{-2}$ and $\bar{Q}_{\dot{a}}^{-}=Q_{\dot{a}}^{-1}-i Q_{\dot{a}}^{-2}:^{3}$

$$
\begin{aligned}
& {\left[J^{i j}, Q^{ \pm}\right]=\frac{i}{2} \gamma^{i j} Q^{ \pm}, \quad\left[J^{i^{\prime} j^{\prime}}, Q^{ \pm}\right]=\frac{i}{2} \gamma^{i^{\prime} j^{\prime}} Q^{ \pm},} \\
& {\left[J^{+I}, Q^{-}\right]=-\frac{i}{2} \gamma^{I} Q^{+},} \\
& {\left[P^{I}, Q^{-}\right]=\frac{\mu}{2 p^{+}} \gamma^{I} \Pi Q^{+}, \quad\left[H, Q^{+}\right]=-\frac{\mu}{2 p^{+}} \Pi Q^{+},}
\end{aligned}
$$

together with the commutators that follow from these by complex conjugation and

$$
\begin{aligned}
& \left\{Q_{a}^{+}, \bar{Q}_{b}^{+}\right\}=\delta_{a b} 2 P^{+} \\
& \left\{Q_{a}^{+}, \bar{Q}_{\dot{a}}^{-}\right\}=\gamma_{a \dot{a}}^{I} P^{I}+i \frac{\mu}{p^{+}}\left(\Pi \gamma^{I}\right)_{a \dot{a}} J^{+I}, \\
& \left\{Q_{\dot{a}}^{-}, \bar{Q}_{\dot{b}}^{-}\right\}=\delta_{\dot{a} \dot{b}} 2 H-i \frac{\mu}{2 p^{+}}\left(\gamma^{i j} \Pi\right)_{\dot{a} \dot{b}} J^{i j}+i \frac{\mu}{2 p^{+}}\left(\gamma^{i^{\prime} j^{\prime}} \Pi\right)_{\dot{a} \dot{b}} J^{i^{\prime} j^{\prime}} .
\end{aligned}
$$

\footnotetext{
${ }^{3}$ For the commutation relations between even generators, see $[2,3]$.
} 
The supersymmetry algebra (3.23) is particularly of importance in describing string interactions in light-cone string field theory formalism [13]. Note that the supersymmetry algebra in (3.23) distinguishes the two $S O(4)$ directions with opposite sign. ${ }^{4}$ This sign flip may be read off from Eq. (2.13) in [2] or Eq. (2.59) in [3] since $\Pi_{\dot{a} \dot{b}}=-\Pi_{\dot{a} \dot{b}}^{\prime}$ in the space with $S O(8)$ negative chirality as explained in Appendix A. The dynamical supersymmetry algebra in Eq. (3.23) is consistent with the $Z_{2}$ symmetry which interchanges simultaneously the two $S O(4)$ directions [18]

$$
Z_{2}:\left(x^{1}, x^{2}, x^{3}, x^{4}\right) \leftrightarrow\left(x^{5}, x^{6}, x^{7}, x^{8}\right)
$$

This should be the case since the worldsheet string action (2.1) and the hamiltonian $H$ are $Z_{2}$ invariant. Indeed the plane wave supergravity spectrums in [3] explicitly respect this $Z_{2}$ symmetry (see also footnote 2 in [18]). The presence of the relative sign in Eq. (3.23) is different from $[13,20]$ although these parts are not corrected by the interaction, so do not affect their results.

\subsection{Open string}

In the presence of D-brane of type $(m, n)$ in the plane wave background $(1.1)$, the symmetries of open string on the D-branes are further broken by the boundary conditions of the open string. First of all, the translation and the boost to the transverse directions of D-brane, generated by $P^{r^{\prime}}$ and $J^{+r^{\prime}}$, are no longer the symmetries of open string modes. Furthermore the rotational symmetry $S O(4) \times S O(4)^{\prime}$ is more broken to $S O(m) \times S O(4-m) \times S O(n) \times S O(4-n)$. Thus we will get $J^{r s^{\prime}}=0$ as expected. ${ }^{5}$

The supersymmetry breaking is more complicated depending on a specific boundary condition, that is, $D_{ \pm}$boundary conditions. It was shown in $[28,29]$ that $D_{-}$-branes preserve 8 kinematical and 8 dynamical supersymmetries and $D_{+}$-branes preserve 8 kinematical supersymmetries regardless of location. However, it was conjectured in [30] that, among $D_{+}$-branes, D1-branes and D5-branes of type $(4,0)$ or $(0,4)$ only preserve 8 dynamical supersymmetries. Here we will give a worldsheet derivation for this conjecture.

Since the open string action is just defined by the closed string action imposed the open string boundary conditions (2.15) and (2.17), the super-Nöther charges of an open string will be given by a subset of the symmetries of the closed string action which are compatible with the open string boundary conditions. Due to the boundary condition (2.17), it turns out that

\footnotetext{
${ }^{4}$ In order to derive Eq. (3.23), one may use the formula, (A.14), (A.17), and (A.19). The relative sign is indeed due to Eqs. (A.17) and (A.19).

${ }^{5}$ However, the translation and the boost generated by $P^{r^{\prime}}$ and $J^{+r^{\prime}}$ are still the symmetries of the action (2.1) and the isometry of the target spacetime. Thus the symmetry transformation by the broken generators, $P^{r^{\prime}}$ and $J^{+r^{\prime}}$ as well as $J^{r s^{\prime}}$, results in new D-branes, symmetry related D-branes, which are in general time-dependent branes. See $[28,29]$ for a detailed discussion on the symmetry related D-branes.
} 
the conserved dynamical supercharge is given by the combination

$$
q^{-}=Q^{-2}-\Omega^{T} Q^{-1}
$$

or equivalently,

$$
q^{-}=Q^{-1}-\Omega Q^{-2}
$$

Using the equations of motion, Eqs. (2.4) and (2.5), it is not difficult to show that the dynamical supercharge density $q_{\tau}^{-}$in Eq. (3.25) satisfies the following conservation law

$$
\frac{\partial q_{\tau}^{-}}{\partial \tau}=-\frac{\partial q_{\sigma}^{-}}{\partial \sigma}
$$

where

$$
\begin{aligned}
q_{\sigma}^{-}= & \sqrt{\frac{1}{2 p^{+}}}\left(\left(\partial_{\tau} X^{r} \gamma^{r} \Omega^{T}+\mu X^{r} \gamma^{r} \Pi\right)\left(S^{1}-\Omega S^{2}\right)-\left(\partial_{\tau} X^{r^{\prime}} \gamma^{r^{\prime}} \Omega^{T}-\mu X^{r^{\prime}} \gamma^{r^{\prime}} \Pi\right)\left(S^{1}+\Omega S^{2}\right)\right. \\
& \left.-\partial_{\sigma} X^{r} \gamma^{r} \Omega^{T}\left(S^{1}+\Omega S^{2}\right)+\partial_{\sigma} X^{r^{\prime}} \gamma^{r^{\prime}} \Omega^{T}\left(S^{1}-\Omega S^{2}\right)\right)
\end{aligned}
$$

for $D_{-}$-branes and

$$
\begin{aligned}
q_{\sigma}^{-}= & \sqrt{\frac{1}{2 p^{+}}}\left(-\left(\partial_{\sigma} X^{r} \gamma^{r} \Omega^{T}-\mu X^{r} \gamma^{r} \Pi\right)\left(S^{1}+\Omega S^{2}\right)+\left(\partial_{\sigma} X^{r^{\prime}} \gamma^{r^{\prime}} \Omega^{T}+\mu X^{r^{\prime}} \gamma^{r^{\prime}} \Pi\right)\left(S^{1}-\Omega S^{2}\right)\right. \\
& \left.+\partial_{\tau} X^{r} \gamma^{r} \Omega^{T}\left(S^{1}-\Omega S^{2}\right)-\partial_{\tau} X^{r^{\prime}} \gamma^{r^{\prime}} \Omega^{T}\left(S^{1}+\Omega S^{2}\right)\right)
\end{aligned}
$$

for $D_{+}$-branes. The open string boundary conditions, (2.15) and (2.17), imply that, in the case of $D_{-}$-branes,

$$
\left.q_{\sigma}^{-}\right|_{\partial \Sigma}=\left.\sqrt{\frac{2}{p^{+}}} \mu\left(X^{r^{\prime}} \gamma^{r^{\prime}} \Pi S^{1}\right)\right|_{\partial \Sigma}
$$

while, for $D_{+}$-branes,

$$
\left.q_{\sigma}^{-}\right|_{\partial \Sigma}=\left.\sqrt{\frac{2}{p^{+}}}\left(\partial_{\sigma} X^{r} \gamma^{r} \Omega^{T} S^{1}-\mu X^{r} \gamma^{r} \Pi S^{1}\right)\right|_{\partial \Sigma}
$$

Thus, in order for the dynamical supercharge $q^{-}=\frac{1}{\pi|\alpha|} \int d \sigma q_{\tau}^{-}$in Eq. (3.25) or Eq. (3.26) to be conserved, the Dirichlet coordinates of $D_{-}$-branes should satisfy

$$
\left.X^{r^{\prime}}\right|_{\partial \Sigma}=0, \quad \forall r^{\prime}
$$

and the Neumann coordinates of $D_{+}$-branes should satisfy

$$
\left.\left(\partial_{\sigma} X^{r} \Omega^{T} S^{1}-\mu X^{r} \Pi S^{1}\right)\right|_{\partial \Sigma}=0, \quad \forall r .
$$

One can see from Eq. (3.32) that $D_{-}$-branes located at a constant transverse position $x_{0}^{r^{\prime}}$ superficially appear to break all dynamical supersymmetries, and the violating terms vanish 
when the transverse position is set to zero, $x_{0}^{r^{\prime}}=0$. However it can be shown [28] that the broken dynamical supersymmetries can be restored by incorporating a worldsheet symmetry realized in the action (2.1). We will explain along the line given in [28] how to use the worldsheet symmetry to restore the dynamical supersymmetry.

The superstring action (2.1) in light-cone gauge is quadratic in the fields. This peculiar property implies that it is invariant up to boundary terms under a shift of the field by a parameter that satisfies the free field equation. For definiteness, let us consider an arbitrary shift of the fields

$$
\delta X^{I}(\sigma, \tau)=\eta^{I}(\sigma, \tau), \quad \delta S^{A}(\sigma, \tau)=\epsilon^{A}(\sigma, \tau) .
$$

Under the transformation, the action (2.1) changes as follows

$$
\begin{aligned}
\delta S= & -\frac{1}{2 \pi \alpha^{\prime} p^{+}} \int d \tau \int_{0}^{2 \pi \alpha^{\prime}\left|p^{+}\right|} d \sigma\left(X^{I}\left(\partial_{+} \partial_{-} \eta^{I}+\mu^{2} \eta^{I}\right)+2 i \bar{S}\left(\rho^{A} \partial_{A}-\mu \Pi\right) \epsilon\right) \\
& -\frac{1}{2 \pi \alpha^{\prime} p^{+}} \int_{\partial \Sigma} d \tau\left(X^{I} \partial_{\sigma} \eta^{I}+i S^{1}\left(\epsilon^{1}-\Omega \epsilon^{2}\right)\right) .
\end{aligned}
$$

For open strings, we can get a new symmetry corresponding to the shift of the fields, Eq. (3.34), if the parameters $\eta^{I}$ and $\epsilon^{A}$ satisfy the free field equations

$$
\begin{gathered}
\partial_{+} \partial_{-} \eta^{I}+\mu^{2} \eta^{I}=0, \\
\left(\rho^{A} \partial_{A}-\mu \Pi\right) \epsilon=0,
\end{gathered}
$$

as well as the following boundary conditions ${ }^{6}$

$$
\begin{aligned}
& \left.\partial_{\sigma} \eta^{r}\right|_{\partial \Sigma}=0 \\
& \left.\partial_{\tau} \eta^{r^{\prime}}\right|_{\partial \Sigma}=0 \\
& \left.\epsilon^{1}\right|_{\partial \Sigma}=\left.\Omega \epsilon^{2}\right|_{\partial \Sigma} .
\end{aligned}
$$

Note that both the equations of motion and the above boundary conditions are identical to those satisfied by the original fields. For closed strings, the transformation (3.34) is just a trivial field redefinition.

This mechanism can be used to restore the broken dynamical supersymmetry of $D_{-}$-branes located away from the origin. In this case one can combine a closed string transformation with a transformation of the form (3.34) to obtain a good symmetry of the open string. In this way one can find modified transformation rules by the use of worldsheet symmetries that lead to a conserved charge [28, 29]. Taking into account the extra worldsheet symmetry, the on-shell conserved current is actually the same as the current for the brane at origin, but with $X^{r^{\prime}}(\sigma, \tau)$

\footnotetext{
${ }^{6}$ For D5-brane case with the boundary term (2.37), the boundary variation for Nuemann coordinates is shifted as $\partial_{\sigma} \eta^{r} \rightarrow \partial_{\sigma} \eta^{r}-\mu \eta^{r}$ and the boundary condition is instead $\left.\partial_{\sigma} \eta^{r}\right|_{\partial \Sigma}=\left.\mu \eta^{r}\right|_{\partial \Sigma}$.
} 
replaced by $\left(X^{r^{\prime}}(\sigma, \tau)-x_{0}^{r^{\prime}}(\sigma)\right)$ where $x_{0}^{r^{\prime}}(\sigma)$ is given by Eq. (2.27). Thus the corresponding charge expressed in terms of oscillators is exactly the same as that for the brane at the origin. This immediately implies that the supersymmetry algebras are also the same.

On the other hand, one can not use the worldsheet symmetry to restore some apparently broken dynamical supersymmetry of $D_{+}$-branes since the symmetry breaking terms are now involved with Neumann coordinates as seen in Eq. (3.33). ${ }^{7}$ Only special classes of $D_{+}$-branes allow the condition (3.33). These are D1-branes in which, by definition, $X^{r}=0$ for all $r$ and D5branes of type $(4,0)$ or $(0,4)$ with the Born-Infeld flux $(2.38)$ where $\Omega^{T} S^{1}=\Pi S^{1}$. Remaining $D_{+}$-branes can not satisfy the condition (3.33) and thus the dynamical supersymmetry is not conserved.

Let us decompose a generic field $\Psi(\sigma, \tau)$ into a zero mode part $\Psi_{0}(\sigma, \tau)$ and a non-zero mode part $\widetilde{\Psi}(\sigma, \tau)$. As one can see from the explicit mode expansions in Sec. 2, the zero mode part $\Psi_{0}(\sigma, \tau)$ separately satisfies the field equations, Eqs. (2.4)-(2.5), and the boundary conditions, Eqs. (2.15)-(2.17). Similar consideration applied to Eq. (3.35) thus leads to

$$
S[\Psi(\sigma, \tau)]=S[\widetilde{\Psi}(\sigma, \tau)]
$$

Since we are interested only in the on-shell values of super-Nöther charges, Eq. (3.41) implies that a Nöther charge $J^{G}$ can also be decomposed into a zero mode part $J_{0}^{G}$ and a non-zero mode part $\widetilde{J}^{G}$, that is $J^{G}=J_{0}^{G}+\widetilde{J}^{G}$. In particular the super Nöther charges $\widetilde{J}^{G}$ can be obtained by applying the same Nöther method to $S[\widetilde{\Psi}(\sigma, \tau)]$, which just gives the same expressions with replacement, $\Psi(\sigma, \tau) \rightarrow \widetilde{\Psi}(\sigma, \tau)$. After we isolate zero mode parts in this way which possibly contain non-periodic functions, we can apply a periodic doubling of open strings [37] for the remaining non-zero mode parts since they contain only periodic functions. The doubling trick will be useful in the actual calculation.

\subsubsection{D_-brane}

In order to derive the super-Nöther charges in terms of open string modes, we will use a proper doubling of the interval $[0, \pi|\alpha|]$ to $[0,2 \pi|\alpha|]$, as done in [23], such that all the classical solutions satisfy the open string boundary conditions for the interval $[0, \pi|\alpha|]$ and periodic boundary conditions for $[0,2 \pi|\alpha|]$. The hamiltonian $H$ for a D-brane located away from the origin does depend on the Dirichlet zero modes $x_{0}^{r^{\prime}}(\sigma)$, so we first isolate the part, denoted as $\Delta H$, for the reason explained above and directly calculate it:

$$
\begin{aligned}
2 p^{+} \Delta H & =\frac{1}{2 \pi \alpha} \int_{0}^{\pi|\alpha|} d \sigma\left(x_{0 r^{\prime}}^{\prime 2}+\mu^{2} x_{0 r^{\prime}}^{2}\right) \\
& =\frac{\mu}{\pi \alpha} \frac{e^{\pi \mu|\alpha|}-1}{e^{\pi \mu|\alpha|}+1} x_{0 r^{\prime}}^{2}
\end{aligned}
$$

\footnotetext{
${ }^{7}$ An essential difference between a Dirichlet coordinate $X^{r^{\prime}}(\sigma, \tau)$ and a Neumann coordinate $X^{r}(\sigma, \tau)$ is that $\left.X^{r^{\prime}}\right|_{\partial \Sigma}$ at boundary depends only on zero modes $x_{0}^{r^{\prime}}$, but $\left.X^{r}\right|_{\partial \Sigma}$ does on all modes, as proved by Eq. (2.26).
} 
For remaining parts, we will use the doubling trick.

It is then straightforward to get the expressions of the super-Nöther charges in terms of the mode expansions in Eqs. (2.26) and (2.28). The result is [23]

$$
\begin{aligned}
& P^{+}=p^{+}, \quad P^{r}=p_{0}^{r}, \quad J^{+r}=-x_{0}^{r} p^{+}, \\
& Q^{+}=\sqrt{2 p^{+}}\left(1+i \Omega^{T}\right) S_{0}, \quad \bar{Q}^{+}=\sqrt{2 p^{+}}\left(1-i \Omega^{T}\right) S_{0}, \\
& J^{r s}=x_{0}^{r} p_{0}^{s}-x_{0}^{s} p_{0}^{r}-i S_{0} \gamma^{r s} S_{0}-i \sum_{n \neq 0}\left\{\frac{1}{2 \omega_{n}}\left(\alpha_{-n}^{r} \alpha_{n}^{s}-\alpha_{-n}^{s} \alpha_{n}^{r}\right)+S_{-n} \gamma^{r s} S_{n}\right\}, \\
& J^{r^{\prime} s^{\prime}}=-i S_{0} \gamma^{r^{\prime} s^{\prime}} S_{0}-i \sum_{n \neq 0}\left\{\frac{1}{2 \omega_{n}}\left(\alpha_{-n}^{r^{\prime}} \alpha_{n}^{s^{\prime}}-\alpha_{-n}^{s^{\prime}} \alpha_{n}^{r^{\prime}}\right)+S_{-n} \gamma^{r^{\prime} s^{\prime}} S_{n}\right\}, \\
& 2 p^{+} H=2 p^{+} \Delta H+\frac{1}{2}\left(p_{0 r}^{2}+\mu^{2} x_{0 r}^{2}\right)-2 \mu i S_{0} \Omega \Pi S_{0}+\sum_{n \neq 0}\left\{\frac{1}{2} \alpha_{-n}^{I} \alpha_{n}^{I}+2 \omega_{n} S_{-n} S_{n}\right\}, \\
& \sqrt{2 p^{+}} Q^{-1}=p_{0}^{r} \gamma^{r} S_{0}+\mu x_{0}^{r} \gamma^{r} \Omega \Pi S_{0}-\sum_{n \neq 0}\left\{c_{n} \alpha_{-n}^{I} \Omega \gamma^{I} S_{n}-\frac{i \mu}{2 c_{n} \omega_{n}} \alpha_{-n}^{I} \gamma^{I} \Pi S_{n}\right\}, \\
& \sqrt{2 p^{+}} Q^{-2}=p_{0}^{r} \gamma^{r} \Omega^{T} S_{0}+\mu x_{0}^{r} \gamma^{r} \Pi S_{0}+\sum_{n \neq 0}\left\{c_{n} \alpha_{-n}^{I} \gamma^{I} S_{n}-\frac{i \mu}{2 c_{n} \omega_{n}} \alpha_{-n}^{I} \Omega^{T} \gamma^{I} \Pi S_{n}\right\},
\end{aligned}
$$

where $J^{r s} \in S O(m)$ or $S O(n)$ and $J^{r^{\prime} s^{\prime}} \in S O(4-m)$ or $S O(4-n)$. Note that $Q^{+}$and $\bar{Q}^{+}$as well as $Q^{-1}$ and $Q^{-2}$ are not independent of but are related to each other since

$$
Q^{+}+\bar{Q}^{+}+i \Omega\left(Q^{+}-\bar{Q}^{+}\right)=0, \quad Q^{-1}+\Omega Q^{-2}=0 .
$$

Thus we take the following independent supercharges, which are preserved supersymmetries for D_-branes as shown before,

$$
\begin{aligned}
& q^{+}=\frac{1}{2}\left(Q^{+}+\bar{Q}^{+}-i \Omega\left(Q^{+}-\bar{Q}^{+}\right)\right)=2 \sqrt{2 p^{+}} S_{0}, \\
& q^{-}=Q^{-2}-\Omega^{T} Q^{-1}=2 Q^{-2} .
\end{aligned}
$$

Similarly we present only the non-vanishing (anti-)commutation relations involved with the odd generators, $q^{ \pm}$:

$$
\begin{aligned}
& {\left[J^{r s}, q^{ \pm}\right]=\frac{i}{2} \gamma^{r s} q^{ \pm}, \quad\left[J^{r^{\prime} s^{\prime}}, q^{ \pm}\right]=\frac{i}{2} \gamma^{r^{\prime} s^{\prime}} q^{ \pm},} \\
& {\left[J^{+r}, q^{-}\right]=\frac{i}{2} \Omega^{T} \gamma^{r} q^{+},} \\
& {\left[P^{r}, q^{-}\right]=-i \frac{\mu}{2 p^{+}} \gamma^{r} \Pi q^{+}, \quad\left[H, q^{+}\right]=i \frac{\mu}{2 p^{+}} \Omega \Pi q^{+},} \\
& \left\{q_{a}^{+}, q_{b}^{+}\right\}=\delta_{a b} 2 P^{+}, \\
& \left\{q_{a}^{+}, q_{\dot{a}}^{-}\right\}=\left(\Omega \gamma^{r}\right)_{a \dot{a}} P^{r}-\frac{\mu}{p^{+}}\left(\Pi \gamma^{r}\right)_{a \dot{a}} J^{+r},
\end{aligned}
$$




$$
\begin{aligned}
\left\{q_{\dot{a}}^{-}, q_{\dot{b}}^{-}\right\}= & \delta_{\dot{a} \dot{b}} 2(H-\Delta H)+\frac{\mu}{2 p^{+}}\left(\left(\gamma_{I}^{r s} \Pi \Omega\right)_{\dot{a} \dot{b}} J_{I}^{r s}+\left(\gamma_{I}^{r^{\prime} s^{\prime}} \Pi \Omega\right)_{\dot{a} \dot{b}} J_{I}^{r^{\prime} s^{\prime}}\right) \\
& -\frac{\mu}{2 p^{+}}\left(\left(\gamma_{I I}^{r s} \Pi \Omega\right)_{\dot{a} \dot{b}} J_{I I}^{r s}+\left(\gamma_{I I}^{r^{\prime} s^{\prime}} \Pi \Omega\right)_{\dot{a} \dot{b}} J_{I I}^{r^{\prime} s^{\prime}}\right)
\end{aligned}
$$

where $J_{I}^{r s} \in S O(m), J_{I}^{r^{\prime} s^{\prime}} \in S O(4-m), J_{I I}^{r s} \in S O(n)$, and $J_{I I}^{r^{\prime} s^{\prime}} \in S O(4-n)$. Note that the open string supersymmetry algebra in (3.58) also distinguishes the two $S O(4)$ directions with relative sign. The supersymmetry algebra (3.58) shows that the BPS bound is saturated by the states with energy $\Delta H$. This implies that the brane located away from the origin does not tend to move towards the origin [29].

\subsection{2 $D_{+}$-brane}

As identified by Skenderis and Taylor $[28,29]$, the open strings on $D_{+}$-brane preserve a different kind of kinematical supersymmetries not descending from the closed string. ${ }^{8}$ The conserved Nöther current is

$$
\begin{aligned}
& q_{\tau}^{+}=\sqrt{2 p^{+}} \sqrt{\frac{\pi \mu|\alpha|}{\sinh \pi \mu|\alpha|}} e^{\mu\left(\sigma-\frac{1}{2} \pi|\alpha|\right) \Omega \Pi}\left(S^{1}+\Omega S^{2}\right), \\
& q_{\sigma}^{+}=\sqrt{2 p^{+}} \sqrt{\frac{\pi \mu|\alpha|}{\sinh \pi \mu|\alpha|}} e^{\mu\left(\sigma-\frac{1}{2} \pi|\alpha|\right) \Omega \Pi}\left(S^{1}-\Omega S^{2}\right) .
\end{aligned}
$$

It is simple to check that

$$
\frac{\partial q_{\tau}^{+}}{\partial \tau}+\frac{\partial q_{\sigma}^{+}}{\partial \sigma}=0
$$

using the equations of motion (2.5) and that

$$
\left.q_{\sigma}^{+}\right|_{\partial \Sigma}=0
$$

This together with the current conservation implies that the charge

$$
q^{+}=\frac{1}{\pi|\alpha|} \int_{0}^{\pi|\alpha|} d \sigma q_{\tau}^{+}
$$

is conserved and in particular all non-zero modes cancel against each other. In Appendix C, we will directly derive the kinematical supercurrent (3.59) from the open string mode expansion.

Using the expression for the supercharges of closed string, Eqs. (3.8)-(3.9), we showed that half of the dynamical supersymmetries are preserved by D1-branes and D5-branes of type $(4,0)$ or $(0,4)$ only. For the supersymmetric $D_{+}$-branes, the $\Omega$ matrices in Eq. (A.16) are the following:

$$
\begin{aligned}
& \text { D1-brane of type }(0,0): \Omega_{a b}=\delta_{a b}, \quad \Omega_{\dot{a} \dot{b}}=\delta_{\dot{a} \dot{b}}, \\
& \text { D5-brane of type }(4,0): \Omega_{a b}=\Pi_{a b}=\Pi_{a b}^{\prime}, \quad \Omega_{\dot{a} \dot{b}}=\Pi_{\dot{a} \dot{b}}=-\Pi_{\dot{a} \dot{b}}^{\prime}, \\
& \text { D5-brane of type }(0,4): \Omega_{a b}=\Pi_{a b}^{\prime}=\Pi_{a b}, \quad \Omega_{\dot{a} \dot{b}}=\Pi_{\dot{a} \dot{b}}^{\prime}=-\Pi_{\dot{a} \dot{b}} .
\end{aligned}
$$

\footnotetext{
${ }^{8}$ We thank K. Skenderis and M. Taylor for related discussions.
} 
Since the nature of the mode expansion depends sensitively on the particular branes on which the open string terminates, we will discuss the supersymmetry algebra case by case. First we will discuss D1-brane. Since the mode expansion of bosonic fields in this case is exactly the same as that of $D_{-}$-branes except for $X^{r}(\sigma, \tau)=0$, it is sufficient to newly calculate the fermionic parts only. Using the methodology mentioned in Eq. (3.41), it is straightforward to calculate the hamiltonian for D1-brane and the result is

$$
2 p^{+} H=2 p^{+} \Delta H+\sum_{n \neq 0}\left\{\frac{1}{2} \alpha_{-n}^{I} \alpha_{n}^{I}+2 \omega_{n} S_{-n} S_{n}\right\},
$$

Note that the open string mass term, $S_{0} \Omega \Pi S_{0}$, is absent, so the ground states form a degenerate supermultiplet while the ground state of the open string for $D_{-}$-brane is an unmatched boson due to the mass term. Similarly, one can obtain the mode expansion for the rotation generators:

$$
J^{r^{\prime} s^{\prime}}=-i \widehat{S}_{0} \gamma^{r^{\prime} s^{\prime}} \widehat{S}_{0}-i \sum_{n \neq 0}\left\{\frac{1}{2 \omega_{n}}\left(\alpha_{-n}^{r^{\prime}} \alpha_{n}^{s^{\prime}}-\alpha_{-n}^{s^{\prime}} \alpha_{n}^{r^{\prime}}\right)+S_{-n} \gamma^{r^{\prime} s^{\prime}} S_{n}\right\},
$$

where $\widehat{S}_{0}$ is defined by Eq. (C.3) and their anti-commutation relation is given by Eq. (C.4).

The conserved dynamical supersymmetry is given by (3.25) and, in the case of D1-brane, it is of the form

$$
\begin{aligned}
\sqrt{2 p^{+}} q^{-} & =\sqrt{2 p^{+}}\left(Q^{-2}-Q^{-1}\right) \\
& =\frac{1}{\pi|\alpha|} \int_{0}^{\pi|\alpha|} d \sigma\left(\dot{X}_{I} \gamma^{I}\left(S^{2}-S^{1}\right)+X_{I}^{\prime} \gamma^{I}\left(S^{1}+S^{2}\right)+\mu X_{I} \gamma^{I} \Pi\left(S^{1}+S^{2}\right)\right)
\end{aligned}
$$

This expression coincides with $[28,29,30]$. The explicit mode expansion of the supercharge (3.69) is then found to be

$$
\begin{aligned}
\sqrt{2 p^{+}} q^{-}= & 2 \mu \sqrt{\frac{2 \tanh \frac{\pi}{2} \mu|\alpha|}{\pi \mu|\alpha|}} x_{0}^{r^{\prime}} \gamma^{r^{\prime}} \widehat{S}_{0} \\
& +\sum_{n \neq 0}\left(c_{n} \alpha_{-n}^{r^{\prime}} \gamma^{r^{\prime}}\left(S_{n}+\widetilde{S}_{n}\right)-\frac{i \mu}{2 c_{n} \omega_{n}} \alpha_{-n}^{r^{\prime}} \gamma^{r^{\prime}} \Pi\left(S_{n}-\widetilde{S}_{n}\right)\right) .
\end{aligned}
$$

Note that the dynamical supersymmetries in this case are preserved regardless of transverse location [28].

The supersymmetry algebra for D1-brane is closed where $P^{r}=J^{+r}=0$ identically:

$$
\begin{aligned}
& {\left[J^{r^{\prime} s^{\prime}}, q^{ \pm}\right]=\frac{i}{2} \gamma^{r^{\prime} s^{\prime}} q^{ \pm}} \\
& {\left[H, q^{ \pm}\right]=0} \\
& \left\{q_{a}^{+}, q_{b}^{+}\right\}=\delta_{a b} 2 P^{+} \\
& \left\{q_{a}^{+}, q_{\dot{a}}^{-}\right\}=\sqrt{2 p^{+}} \sqrt{\frac{2 \mu \tanh \frac{\pi}{2} \mu|\alpha|}{\pi|\alpha|}} x_{0}^{r^{\prime}} \gamma_{a \dot{a}}^{r^{\prime}}, \\
& \left\{q_{\dot{a}}^{-}, q_{\dot{b}}^{-}\right\}=\delta_{\dot{a} \dot{b}} 2 H .
\end{aligned}
$$


Now we will consider the D5-brane of type $(0,4)$ for definiteness which presumably represents the Penrose limit of a baryon vertex (the $(4,0)$ brane can be treated similarly). In this case the boundary term (2.37) contributes to the hamiltonian which is now given by

$$
2 p^{+} H=\frac{1}{\pi|\alpha|} \int_{0}^{\pi|\alpha|} d \sigma\left[\frac{1}{2} \dot{X}_{r}^{2}+\frac{1}{2}\left(\partial_{\sigma} X_{r}-\mu X_{r}\right)^{2}+\frac{1}{2}\left(\dot{X}_{r^{\prime}}^{2}+X_{r^{\prime}}^{\prime 2}+\mu^{2} X_{r^{\prime}}^{2}\right)+i S^{A} \dot{S}^{A}\right] .
$$

Since the mode expansion in this case is essentially the same as the case of D1-brane except for the Neumann coordinates, $X^{r}(\sigma, \tau)$ in Eq. (2.40), it is sufficient to newly calculate the parts involved with the Neumann coordinates. The result for the hamiltonian is

$$
2 p^{+} H=2 p^{+} \Delta H+\frac{1}{2} p_{0 r}^{2}+\sum_{n \neq 0}\left\{\frac{1}{2} \alpha_{-n}^{I} \alpha_{n}^{I}+2 \omega_{n} S_{-n} S_{n}\right\} .
$$

Similarly, for the rotation generators, we get

$$
\begin{aligned}
& J^{r s}=x_{0}^{r} p_{0}^{s}-x_{0}^{s} p_{0}^{r}-i \widehat{S}_{0} \gamma^{r s} \widehat{S}_{0}-i \sum_{n \neq 0}\left\{\frac{1}{2 \omega_{n}}\left(\alpha_{-n}^{r} \alpha_{n}^{s}-\alpha_{-n}^{s} \alpha_{n}^{r}\right)+S_{-n} \gamma^{r s} S_{n}\right\}, \\
& J^{r^{\prime} s^{\prime}}=-i \widehat{S}_{0} \gamma^{r^{\prime} s^{\prime}} \widehat{S}_{0}-i \sum_{n \neq 0}\left\{\frac{1}{2 \omega_{n}}\left(\alpha_{-n}^{r^{\prime}} \alpha_{n}^{s^{\prime}}-\alpha_{-n}^{s^{\prime}} \alpha_{n}^{r^{\prime}}\right)+S_{-n} \gamma^{r^{\prime} s^{\prime}} S_{n}\right\} .
\end{aligned}
$$

For open strings on D5-brane with the Born-Infeld flux (2.38), the preserved dynamical supersymmetry is $q^{-}=\left(Q^{-2}-\Omega^{T} Q^{-1}\right)$. For the $(0,4)$ brane, for example, the dynamical supercharge is given by

$$
\begin{aligned}
\sqrt{2 p^{+}} q^{-}= & \sqrt{2 p^{+}}\left(Q^{-2}+\Pi Q^{-1}\right) \\
= & 2 p_{0}^{r} \gamma^{r} \Pi \widehat{S}_{0}+2 \mu \sqrt{\frac{2 \tanh \frac{\pi}{2} \mu|\alpha|}{\pi \mu|\alpha|}} x_{0}^{r^{\prime}} \gamma^{r^{\prime}} \Pi \widehat{S}_{0} \\
& +2 \sum_{n \neq 0}\left\{c_{n} \alpha_{-n}^{I} \gamma^{I} S_{n}+\frac{i \mu}{2 c_{n} \omega_{n}} \alpha_{-n}^{I} \Pi \gamma^{I} \Pi S_{n}\right\} .
\end{aligned}
$$

The supersymmetry algebra is closed as in D1-brane:

$$
\begin{aligned}
& {\left[J^{r s}, q^{ \pm}\right]=\frac{i}{2} \gamma^{r s} q^{ \pm}, \quad\left[J^{r^{\prime} s^{\prime}}, q^{ \pm}\right]=\frac{i}{2} \gamma^{r^{\prime} s^{\prime}} q^{ \pm},} \\
& {\left[J^{+r}, q^{-}\right]=-\frac{i}{2} \gamma^{r} \Pi q^{+},} \\
& {\left[P^{r}, q^{ \pm}\right]=\left[H, q^{ \pm}\right]=0,} \\
& \left\{q_{a}^{+}, q_{b}^{+}\right\}=\delta_{a b} 2 P^{+}, \\
& \left\{q_{a}^{+}, q_{\dot{a}}^{-}\right\}=\left(\Pi \gamma^{r}\right)_{a \dot{a}} P^{r}+\sqrt{2 p^{+}} \sqrt{\frac{2 \mu \tanh \frac{\pi}{2} \mu|\alpha|}{\pi|\alpha|}} x_{0}^{r^{\prime}}\left(\Pi \gamma^{r^{\prime}}\right)_{a \dot{a}} \\
& \left\{q_{\dot{a}}^{-}, q_{\dot{b}}^{-}\right\}=\delta_{\dot{a} \dot{b}} 2 H .
\end{aligned}
$$


Note that the supersymmetry algebra for $q^{-}$of $D_{+}$-branes does not contain the angular momentum parts and all the supersymmetries commute with the light-cone hamiltonian unlike $D_{-}$-branes. Consequently the vacuum for $D_{+}$-branes is degenerate, containing eight bosons and eight fermions as in flat space, and forms a supermultiplet. The vacuum for $D_{-}$-branes is, however, a singlet under the $q^{-}$supersymmetry and $q^{+}$is a spectrum generating operator.

\section{Discussion}

In this paper we carefully analyzed the supersymmetry algebra of plane wave superstrings. We found that the supersymmetry algebra of closed string respects the $S O(4) \times S O(4)^{\prime} \times Z_{2}$ symmetry where the $Z_{2}$ exchanges the first $S O(4)$ with the second $S O(4)^{\prime}$. We gave a worldsheet derivation for conserved supersymmetries, from which we showed that half of the dynamical supersymmetries are preserved by D1-branes and D5-branes of type $(4,0)$ or $(0,4)$ only among $D_{+}$-branes. In addition we exhaustively analyzed the supersymmetry algebra of open strings on half BPS D-branes, both $D_{-}$-branes and $D_{+}$-branes. We showed that the algebra is closed including a new kind of kinematical supersymmetry restored by incorporating worldsheet symmetry suggested by Skenderis and Taylor [28, 29] and all the supersymmetries for $D_{+}$-branes commute with the light-cone hamiltonian unlike $D_{-}$-branes. Throughout this paper we used the 8-component spinors, $S O(8)$ Majorana-Weyl spinors, so we hope the supersymmetry algebras presented here will be useful for some string field theory calculation in the plane wave background.

In this paper we considered only open strings whose end points are attached on the same D-branes. One may consider open strings connecting Dp-Dp' branes, $\mathrm{p}-\mathrm{p}^{\prime}$ strings, in the plane wave background. The $\mathrm{p}-\mathrm{p}^{\prime}$ string was analyzed in $[25,30]$ by the boundary state description and the computation of cylinder diagrams, mainly for instantonic branes. It will be interesting to explicitly study the supersymmetry algebra of $\mathrm{p}-\mathrm{p}^{\prime}$ strings as done in this paper, since these open strings, especially being BPS states, are dual to a defect conformal field theory in a plane wave background [38, 39, 24]. We will report this result elsewhere [40].

\section{Acknowledgments}

We thank K. Skenderis and M. Taylor for helpful correspondence that allows us to correct an acute error in the preliminary version of this paper. We are also grateful to Kyung-Seok Cha and Chanyong Park for checking several parts of our calculation. BHL thank H. Nicolai and MPI for the hospitality and Nakwoo Kim for helpful discussions. We are supported by the grant from the Interdisciplinary Research Program of the KOSEF (No. R01-1999-00018) and the special grant of Sogang University in 2002. BHL was supported by the Korean Research Foundation Grant KRF D00027. 


\section{Appendix}

\section{A Notations, Definitions and Useful Formula}

The conventions for the indices are:

$I, J, K, \cdots=1, \cdots, 8: S O(8)$ vector indices,

$a, b, c, \cdots=1, \cdots, 8: S O(8)$ spinor indices with positive chirality,

$\dot{a}, \dot{b}, \dot{c}, \cdots=1, \cdots, 8: S O(8)$ spinor indices with negative chirality,

$i, j, k, \cdots=1, \cdots, 4: S O(4)$ vector indices,

$i^{\prime}, j^{\prime}, k^{\prime}, \cdots=5, \cdots, 8: S O(4)^{\prime}$ vector indices.

$A, B=1,2$ : worldsheet (spinor, vector, etc.) indices.

In this paper we consider a Dp-brane of type $(m, n), m+n=p-1$, with $m$ Neumann directions in $S O(4)$ and $n$ Neumann directions in $S O(4)^{\prime}$. Thus we distinguish the Neumann directions and the Dirichlet directions with indices:

$r, s, t, \cdots=1, \cdots, m, 5, \cdots, 4+n$ : vector indices in Neumann directions,

$r^{\prime}, s^{\prime}, t^{\prime}, \cdots=m+1, \cdots, 4,5+n, \cdots, 8$ : vector indices in Dirichlet directions.

The spacetime metric is $\eta^{\mu \nu}=(-1,+1, \cdots,+1)$ where $\mu, \nu$ are $S O(9,1)$ vector indices. We decompose $X^{\mu}$ into the light-cone and transverse coordinates: $X^{\mu}=\left(X^{+}, X^{-}, X^{I}\right)$ where

$$
X^{ \pm}=\frac{1}{\sqrt{2}}\left(X^{0} \pm X^{9}\right)
$$

The worldsheet metric is $\eta^{A B}=(-1,+1)$ where $A, B=\tau, \sigma$.

We adopt the chiral representation for $S O(9,1)$ Dirac matrices $\Gamma^{\mu}$ used in $[2,3]$

$$
\Gamma^{\mu}=\left(\begin{array}{cc}
0 & \gamma^{\mu} \\
\bar{\gamma}^{\mu} & 0
\end{array}\right)
$$

where the $16 \times 16$ matrices, $\gamma^{\mu}=\left(\gamma^{\mu}\right)^{\alpha \beta}=\left(1, \gamma^{I}, \gamma^{9}\right), \bar{\gamma}^{\mu}=\left(\bar{\gamma}^{\mu}\right)_{\alpha \beta}=\left(-1, \gamma^{I}, \gamma^{9}\right), \quad \alpha, \beta=$ $1, \cdots, 16$, satisfy

$$
\gamma^{\mu} \bar{\gamma}^{\nu}+\gamma^{\nu} \bar{\gamma}^{\mu}=2 \eta^{\mu \nu}=\bar{\gamma}^{\mu} \gamma^{\nu}+\bar{\gamma}^{\nu} \gamma^{\mu}
$$

The $S O(9,1)$ chirality matrix $\Gamma_{11} \equiv \Gamma^{0} \cdots \Gamma^{9}$ is given by

$$
\begin{aligned}
& \Gamma_{11}=\left(\begin{array}{cc}
1_{16} & 0 \\
0 & -1_{16}
\end{array}\right), \\
& \gamma^{0} \bar{\gamma}^{1} \cdots \gamma^{8} \bar{\gamma}^{9}=1_{16}, \\
& \bar{\gamma}^{0} \gamma^{1} \cdots \bar{\gamma}^{8} \gamma^{9}=-1_{16}
\end{aligned}
$$

We further assume the following block decomposition for $\gamma^{I}$

$$
\gamma^{I}=\left(\begin{array}{cc}
0 & \gamma_{a \dot{a}}^{I} \\
\tilde{\gamma}_{\dot{a} a}^{I} & 0
\end{array}\right)
$$


where $8 \times 8$ matrices, $\gamma_{a \dot{a}}^{I}, \tilde{\gamma}_{\dot{a} a}^{I}=\left(\gamma^{I^{T}}\right)_{\dot{a} a}$, satisfy

$$
\begin{aligned}
& \gamma_{a \dot{a}}^{I} \tilde{\gamma}_{\dot{a} b}^{J}+\gamma_{a \dot{a}}^{J} \tilde{\gamma}_{\dot{a} b}^{I}=2 \delta^{I J} \delta_{a b}, \\
& \tilde{\gamma}_{\dot{a} a}^{I} \gamma_{a \dot{a}}^{J}+\tilde{\gamma}_{\dot{a} a}^{J} \gamma_{a \dot{a}}^{I}=2 \delta^{I J} \delta_{\dot{a} \dot{b}} .
\end{aligned}
$$

Since we use $S O(8)$ chiral spinors, we take the $S O(8)$ chirality matrix $\gamma \equiv \gamma^{1} \bar{\gamma}^{2} \cdots \gamma^{7} \bar{\gamma}^{8}=\gamma^{0} \bar{\gamma}^{9}$ as

$$
\gamma=\left(\begin{array}{cc}
1_{8} & 0 \\
0 & -1_{8}
\end{array}\right)
$$

We use the following definitions

$$
\begin{array}{ll}
\Pi_{a b}=\gamma^{1} \tilde{\gamma}^{2} \gamma^{3} \tilde{\gamma}^{4}, & \Pi_{\dot{a} \dot{b}}=\tilde{\gamma}^{1} \gamma^{2} \tilde{\gamma}^{3} \gamma^{4}, \\
\Pi_{a b}^{\prime}=\gamma^{5} \tilde{\gamma}^{6} \gamma^{7} \tilde{\gamma}^{8}, & \Pi_{\dot{a} \dot{b}}^{\prime}=\tilde{\gamma}^{5} \gamma^{6} \tilde{\gamma}^{7} \gamma^{8}
\end{array}
$$

Due to the normalization (A.7), $\Pi_{a b}=\Pi_{a b}^{\prime}, \Pi_{\dot{a} \dot{b}}=-\Pi_{\dot{a} \dot{b}}^{\prime}$. Note that the matrix $\Pi$ is symmetric and traceless and $\Pi^{2}=1$. We define the antisymmetrized products of gamma matrices, e.g.,

$$
\begin{aligned}
& \gamma_{a b}^{I J}=\frac{1}{2}\left(\gamma_{a \dot{a}}^{I} \tilde{\gamma}_{\dot{a} b}^{J}-\gamma_{a \dot{a}}^{J} \tilde{\gamma}_{\dot{a} b}^{I}\right), \\
& \gamma_{\dot{a} \dot{b}}^{I J}=\frac{1}{2}\left(\tilde{\gamma}_{\dot{a} a}^{I} \gamma_{a \dot{b}}^{J}-\tilde{\gamma}_{\dot{a} a}^{J} \gamma_{a \dot{b}}^{I}\right), \\
& \gamma_{a \dot{b}}^{I J K}=\frac{1}{3 !}\left(\gamma_{a \dot{a}}^{I} \tilde{\gamma}_{\dot{a} b}^{J} \gamma_{b \dot{b}}^{K} \pm 5 \text { terms }\right), \\
& \gamma_{\dot{a} \dot{b}}^{I J K}=\frac{1}{3 !}\left(\tilde{\gamma}_{\dot{a} a}^{I} \gamma_{a \dot{b}}^{J} \tilde{\gamma}_{\dot{b} b}^{K} \pm 5 \text { terms }\right) .
\end{aligned}
$$

$\gamma^{I J}$ is an antisymmetric matrix, i.e., $\gamma_{b a}^{I J}=-\gamma_{a b}^{I J}$ while $\gamma_{b a}^{I J K L}=\gamma_{a b}^{I J K L}$. Similarly they are true with dotted indices.

The 16-component spinor $\theta$ is decomposed in terms of the 8-component spinors as

$$
\theta^{\alpha}=\left(\begin{array}{c}
S^{a} \\
Q^{\dot{a}}
\end{array}\right),
$$

where $S^{a}=-\frac{1}{2} \gamma^{+} \bar{\gamma}^{-} \theta$ is a positive chirality spinor and $Q^{\dot{a}}=-\frac{1}{2} \gamma^{-} \bar{\gamma}^{+} \theta$ is a negative chirality spinor. Here $\gamma^{ \pm}$is defined by

$$
\gamma^{ \pm}=\frac{1}{\sqrt{2}}\left(\gamma^{0} \pm \gamma^{9}\right)
$$

In computing the supersymmetry algebra we need to use the Fierz identity. For spinors $S_{1}$ and $S_{2}$ with positive chirality,

$$
S_{1}^{a} S_{2}^{b}=\frac{1}{8} \delta_{a b} S_{1} S_{2}+\frac{1}{16} S^{1} \gamma^{I J} S_{2} \gamma_{a b}^{I J}+\frac{1}{384} S_{1} \gamma^{I J K L} S_{2} \gamma_{a b}^{I J K L}
$$


Also the similar expression is true for negative chirality spinors. Another useful identity [32] is

$$
\begin{aligned}
\gamma_{a \dot{a}}^{I} \gamma_{b \dot{b}}^{J} & =\frac{1}{8}\left(\delta^{I J} \delta_{a b} \delta_{\dot{a} \dot{b}}+\delta_{a b} \gamma_{\dot{a} \dot{b}}^{I J}+\delta_{\dot{a} \dot{b}} \gamma_{a b}^{I J}\right) \\
& +\frac{1}{16} \delta^{I J}\left(\gamma_{a b}^{K L} \gamma_{\dot{a} \dot{b}}^{K L}+\frac{1}{24} \gamma_{a b}^{K L M N} \gamma_{\dot{a} \dot{b}}^{K L M N}\right) \\
& -\frac{1}{8}\left(\gamma_{a b}^{I K} \gamma_{\dot{a} \dot{b}}^{J K}+\gamma_{a b}^{J K} \gamma_{\dot{a} \dot{b}}^{I K}\right) \\
& -\frac{1}{48}\left(\gamma_{a b}^{I K L M} \gamma_{\dot{a} \dot{b}}^{J K L M}+\gamma_{a b}^{J K L M} \gamma_{\dot{a} \dot{b}}^{I K L M}\right) \\
& +\frac{1}{16}\left(\gamma_{a b}^{I J K L} \gamma_{\dot{a} \dot{b}}^{K L}+\gamma_{a b}^{K L} \gamma_{\dot{a} \dot{b}}^{I J K L}\right) .
\end{aligned}
$$

In order to discuss the boundary condition of open strings living on a Dp-brane, it is needed to introduce the matrix $\Omega$ defined by

$$
\Omega_{a b}=\left(\prod_{I \in \mathcal{N}} \gamma^{I}\right)_{a b}, \quad \Omega_{\dot{a} \dot{b}}=\left(\prod_{I \in \mathcal{N}} \gamma^{I}\right)_{\dot{a} \dot{b}},
$$

where $\mathcal{N}$ denotes the set of Neumann directions. Note that $\Omega^{T} \Omega=1$. Thus, for the $D_{-}$-branes, $\Pi \Omega$ is an antisymmetric matrix, i.e., $(\Pi \Omega)^{T}=-\Pi \Omega$, while, for $D_{+}$-branes, $(\Pi \Omega)^{T}=\Pi \Omega$. The following commutation relations are useful in the calculation

$$
\begin{array}{ll}
\left\{\gamma^{i}, \Pi\right\}=0, & {\left[\gamma^{i^{\prime}}, \Pi\right]=0,} \\
\left\{\gamma^{r}, \Omega\right\}=0, & {\left[\gamma^{r^{\prime}}, \Omega\right]=0 .}
\end{array}
$$

We often use the subscripts $I \in S O(4)$ and $I I \in S O(4)^{\prime}$ to distinguish the two different $S O(4)$ directions of Neumann and Dirichlet coordinates, e.g., $\gamma_{I}^{r}, \gamma_{I I}^{r}, \gamma_{I}^{r^{\prime}}$, etc. Using (A.17) and (A.18), the following formula can be derived

$$
\begin{aligned}
& f_{I J} \gamma^{K} \gamma^{I J} \Pi \gamma^{K}=f_{I J} \gamma^{K} \Pi \gamma^{I J} \gamma^{K}=4\left(f_{i j} \gamma^{i j}-f_{i^{\prime} j^{\prime}} \gamma^{i^{\prime} j^{\prime}}\right) \Pi, \\
& \gamma^{t} \gamma_{I}^{r s} \Pi \gamma^{t}=(4-m+n) \gamma_{I}^{r s} \Pi, \quad \gamma^{t^{\prime}} \gamma_{I}^{r^{\prime} s^{\prime}} \Pi \gamma^{t^{\prime}}=(4+m-n) \gamma_{I}^{r^{\prime} s^{\prime}} \Pi, \quad \text { etc. }
\end{aligned}
$$

where $f_{I J}$ is a fermion bilinear.

Finally we list useful integral formula which are used in the calculation of $D_{+}$-branes:

$$
\begin{aligned}
& \int_{0}^{\pi|\alpha|} \cosh \mu \sigma \cos \frac{n \sigma}{|\alpha|} d \sigma=(-)^{n} \frac{\mu \alpha^{2}}{\mu^{2} \alpha^{2}+n^{2}} \sinh \pi \mu|\alpha|, \\
& \int_{0}^{\pi|\alpha|} \sinh \mu \sigma \sin \frac{n \sigma}{|\alpha|} d \sigma=-(-)^{n} \frac{n|\alpha|}{\mu^{2} \alpha^{2}+n^{2}} \sinh \pi \mu|\alpha|, \\
& \int_{0}^{\pi|\alpha|} \cosh \mu \sigma \sin \frac{n \sigma}{|\alpha|} d \sigma=-\frac{n|\alpha|}{\mu^{2} \alpha^{2}+n^{2}}\left((-)^{n} \cosh \pi \mu|\alpha|-1\right), \\
& \int_{0}^{\pi|\alpha|} \sinh \mu \sigma \cos \frac{n \sigma}{|\alpha|} d \sigma=\frac{\mu \alpha^{2}}{\mu^{2} \alpha^{2}+n^{2}}\left((-)^{n} \cosh \pi \mu|\alpha|-1\right) .
\end{aligned}
$$




\section{B (Anti-)Commutation Relations for $D_{+}$-branes}

To determine the anti-commutation relation of the zero modes, Eq. (2.33), let us calculate the anti-commutator for the spinor field $S^{A}(\sigma, \tau)$, Eq. (2.25), using the mode expansion (2.31) and the anti-commutation relations, Eqs. (2.34)-(2.36), for the nonzero modes:

$$
\begin{aligned}
\left\{S^{1 a}(\sigma, \tau), S^{1 b}\left(\sigma^{\prime}, \tau\right)\right\}= & \cosh \mu\left(\sigma+\sigma^{\prime}\right)\left\{S_{0}^{a}, S_{0}^{b}\right\}+\sinh \mu\left(\sigma+\sigma^{\prime}\right)(\Omega \Pi)_{a c}\left\{S_{0}^{c}, S_{0}^{b}\right\} \\
& +\frac{1}{4} \delta_{a b} \sum_{n \in \mathbf{Z}} e^{i \frac{n}{|\alpha|}\left(\sigma-\sigma^{\prime}\right)}-\frac{1}{4} \delta_{a b} \sum_{n \in \mathbf{Z}} \frac{\mu^{2}}{\omega_{n}^{2}} e^{i \frac{n}{|\alpha|}\left(\sigma+\sigma^{\prime}\right)} \\
& -\frac{i}{4}(\Omega \Pi)_{a b} \sum_{n \in \mathbf{Z}} \frac{\mu n /|\alpha|}{\omega_{n}^{2}} e^{i \frac{n}{|\alpha|}\left(\sigma+\sigma^{\prime}\right)}
\end{aligned}
$$

where we assumed the form of $\left\{S_{0}^{a}, S_{0}^{b}\right\}$ as $A \delta_{a b}+B(\Omega \Pi)_{a b}$. Now we will use the following integral representation to evaluate the infinite sums in (B.1):

$$
\begin{aligned}
\sum_{n \in \mathbf{Z}} \frac{\mu^{2}}{\omega_{n}^{2}} e^{i \frac{n}{|\alpha|}\left(\sigma+\sigma^{\prime}\right)} & =-\int_{C} \frac{d z}{1-e^{2 \pi i z}} \frac{\mu^{2} \alpha^{2}}{z^{2}+\mu^{2} \alpha^{2}} e^{i\left(\sigma+\sigma^{\prime}\right) z /|\alpha|} \\
& =\pi \mu|\alpha|\left(\frac{e^{-\mu\left(\sigma+\sigma^{\prime}\right)}}{1-e^{-2 \pi \mu|\alpha|}}-\frac{e^{\mu\left(\sigma+\sigma^{\prime}\right)}}{1-e^{2 \pi \mu|\alpha|}}\right), \\
\sum_{n \in \mathbf{Z}} \frac{\mu n /|\alpha|}{\omega_{n}^{2}} e^{i \frac{n}{|\alpha|}\left(\sigma+\sigma^{\prime}\right)} & =-\int_{C} \frac{d z}{1-e^{2 \pi i z}} \frac{\mu|\alpha| z}{z^{2}+\mu^{2} \alpha^{2}} e^{i\left(\sigma+\sigma^{\prime}\right) z /|\alpha|} \\
& =i \pi \mu|\alpha|\left(\frac{e^{-\mu\left(\sigma+\sigma^{\prime}\right)}}{1-e^{-2 \pi \mu|\alpha|}}+\frac{e^{\mu\left(\sigma+\sigma^{\prime}\right)}}{1-e^{2 \pi \mu|\alpha|}}\right),
\end{aligned}
$$

where the contour $C$ consists of two lines passing infinitesimally above and below the real axis. One can see that the contribution from the zero modes is exactly cancelled by the second and the third sums in (B.1) provided that the anti-commutator of the zero modes is given as in (2.33). Thus we have the anti-commutation relation Eq. (2.25). Similarly, one can check that $\left\{S^{1 a}(\sigma, \tau), S^{2 b}\left(\sigma^{\prime}, \tau\right)\right\}=0$.

Using the commutation relations in Eq. (2.29) and the identities (B.2) and (B.3) together with

$$
\begin{aligned}
\sum_{n \in \mathbf{Z}} \frac{n^{2} / \alpha^{2}}{\omega_{n}^{2}} e^{i \frac{n}{|\alpha|}\left(\sigma+\sigma^{\prime}\right)} & =-\int_{C} \frac{d z}{1-e^{2 \pi i z}} \frac{z^{2}}{z^{2}+\mu^{2} \alpha^{2}} e^{i\left(\sigma+\sigma^{\prime}\right) z /|\alpha|} \\
& =-\pi \mu|\alpha|\left(\frac{e^{-\mu\left(\sigma+\sigma^{\prime}\right)}}{1-e^{-2 \pi \mu|\alpha|}}-\frac{e^{\mu\left(\sigma+\sigma^{\prime}\right)}}{1-e^{2 \pi \mu|\alpha|}}\right),
\end{aligned}
$$

one can also check that $X^{r}(\sigma, \tau)$ in Eq. (2.40) satisfies the quantization rule (2.24). 


\section{Kinematical Supersymmetry for $D_{+}$-branes}

Here we directly derive the kinematical supercurrent (3.59) for $D_{+}$-branes from the open string mode expansion. In section 2, we showed that the fermionic mode expansions (2.31) satisfy the boundary condition (2.17) as well as the equations of motion (2.5) and the anti-commutation relations between the modes are given by Eqs. (2.33) and (2.34). Thus the Nöther charge for the kinematical supersymmetry should be represented by some combination of $S^{1}$ and $S^{2}$ fermions in Eq. (2.31). We now examine whether there can be any combination from $S^{1}$ and $S^{2}$ fermions which reduces to a kinematical supersymmetry generator $q^{+}$satisfying the standard superalgebra

$$
\left\{q_{a}^{+}, q_{b}^{+}\right\}=\delta_{a b} 2 P^{+}
$$

To proceed our argument, first note that the anti-commutation relation in Eq. (2.33) can be rewritten as

$$
\left\{S_{0}^{a}, S_{0}^{b}\right\}=\frac{\pi \mu|\alpha|}{4 \sinh \pi \mu|\alpha|}\left(e^{-\pi \mu|\alpha| \Omega \Pi}\right)_{a b}
$$

and thus a zero mode fermion defined by

$$
\widehat{S}_{0}=\sqrt{\frac{\sinh \pi \mu|\alpha|}{\pi \mu|\alpha|}} e^{\frac{1}{2} \pi \mu|\alpha| \Omega \Pi} S_{0}
$$

satisfies the standard anti-commutation relation

$$
\left\{\widehat{S}_{0}^{a}, \widehat{S}_{0}^{b}\right\}=\frac{1}{4} \delta_{a b}
$$

Thus if the supersymmetry generator $q^{+}$would be given by

$$
q^{+}=2 \sqrt{2 p^{+}} \widehat{S}_{0}
$$

$q^{+}$in (C.5) then satisfies the superalgebra (C.1). Interestingly it turns out that such a supersymmetry generator can be constructed from the fermions in Eq. (2.31).

Notice that the zero mode parts, $S_{0}^{1}(\sigma)$ and $S_{0}^{2}(\sigma)$ of $S^{1}(\sigma, \tau)$ and $S^{2}(\sigma, \tau)$, respectively, are given by

$$
\begin{aligned}
& S_{0}^{1}(\sigma)=\sqrt{\frac{\pi \mu|\alpha|}{\sinh \pi \mu|\alpha|}} e^{\mu\left(\sigma-\frac{1}{2} \pi|\alpha|\right) \Omega \Pi} \widehat{S}_{0}, \\
& S_{0}^{2}(\sigma)=\sqrt{\frac{\pi \mu|\alpha|}{\sinh \pi \mu|\alpha|}} \Omega^{T} e^{\mu\left(\sigma-\frac{1}{2} \pi|\alpha|\right) \Omega \Pi} \widehat{S}_{0} .
\end{aligned}
$$

Since the zero modes $S_{0}^{1}(\sigma)$ and $S_{0}^{2}(\sigma)$ satisfy $S_{0}^{1}(\sigma)=\Omega S_{0}^{2}(\sigma)$, as obviously seen in (C.6), we can deduce the form of the charge density $q_{\tau}^{+}$for the kinematical supersymmetry whose zero mode part would be given by (C.5):

$$
q_{\tau}^{+}=A e^{k \mu\left(\sigma-\frac{1}{2} \pi|\alpha|\right) \Omega \Pi}\left(S^{1}+\Omega S^{2}\right)(\sigma, \tau),
$$


where $k \in \mathbf{Z}$. Note that only $k=0$ case is descending from the closed string. Due to the structure of the integrals, Eqs. (A.21)-(A.24), the most plausible choices are $k=-1,0,1$, or explicitly,

$$
\begin{aligned}
& q_{\tau}^{+}=\sqrt{2 p^{+}} \sqrt{\frac{\sinh \pi \mu|\alpha|}{\pi \mu|\alpha|}} e^{-\mu\left(\sigma-\frac{1}{2} \pi|\alpha|\right) \Omega \Pi}\left(S^{1}+\Omega S^{2}\right), \\
& q_{\tau}^{+}=\sqrt{\frac{\pi \mu|\alpha| p^{+}}{\tanh \frac{1}{2} \pi \mu|\alpha|}}\left(S^{1}+\Omega S^{2}\right), \\
& q_{\tau}^{+}=\sqrt{2 p^{+}} \sqrt{\frac{\pi \mu|\alpha|}{\sinh \pi \mu|\alpha|}} e^{\mu\left(\sigma-\frac{1}{2} \pi|\alpha|\right) \Omega \Pi}\left(S^{1}+\Omega S^{2}\right) .
\end{aligned}
$$

The first two candidates, however, obtain contributions from the non-zero modes and so none of them is conserved. For example, the first choice (C.8) gives us

$$
\begin{aligned}
q^{+}= & 2 \sqrt{2 p^{+}} \widehat{S}_{0}+\frac{4 \sqrt{2 p^{+}}}{\pi} \sqrt{\frac{\sinh \pi \mu|\alpha|}{\pi \mu|\alpha|}} \times \\
& \sum_{n \neq 0} \frac{c_{n}}{\omega_{n}} \frac{\mu n}{\mu^{2} \alpha^{2}+n^{2}}\left(e^{\frac{1}{2} \pi \mu|\alpha| \Omega \Pi}-(-)^{n} e^{-\frac{1}{2} \pi \mu|\alpha| \Omega \Pi}\right)\left(\Pi S_{n}-i \rho_{n} \Omega S_{n}\right) e^{-i \omega_{n} \tau} .
\end{aligned}
$$

Here we used the integral formula (A.21)-(A.24). But the third choice (C.10) precisely gives the conserved kinematical supercharge

$$
q^{+}=2 \sqrt{2 p^{+}} \widehat{S}_{0},
$$

first identified by Skenderis and Taylor [28, 29]. 


\section{References}

[1] M. Blau, J. Figueroa-O'Farrill, C. Hull, and G. Papadopoulos, J. High Energy Phys. 01, 047 (2002), hep-th/0110242; Class. Quant. Grav. 19, L87 (2002), hep-th/0201081.

[2] R. R. Metsaev, Nucl. Phys. B625, 70 (2002), hep-th/0112044.

[3] R. R. Metsaev and A. A. Tseytlin, Phys. Rev. D65, 126004 (2002), hep-th/0202109.

[4] D. Berenstein, J. Maldacena and H. Nastase, J. High Energy Phys. 04, 013 (2002), hep-th/0202021.

[5] C. Kristjansen, J. Plefka, G. W. Semenoff, and M. Staudacher, Nucl. Phys. B643, 3 (2002), hep-th/0205033.

[6] D. J. Gross, A. Mikhailov, and R. Roiban, Ann. Phys. 30, 31 (2002), hep-th/0205066.

[7] N. R. Constable, D. Z. Freedman, M. Headrick, S. Minwalla, L. Motl, A. Postnikov, and W. Skiba, J. High Energy Phys. 07, 017 (2002), hep-th/0205089.

[8] C.-S. Chu, V. V. Khoze, and G. Travaglini, J. High Energy Phys. 06, 011 (2002), hep-th/0206005.

[9] A. Santambrogio and D. Zanon, Phys. Lett. B545, 425 (2002), hep-th/0206079.

[10] N. Beisert, C. Kristjansen, J. Plefka, G. W. Semenoff, and M. Staudacher, Nucl. Phys. B650, 125 (2003), hep-th/0208178.

[11] N. R. Constable, D. Z. Freedman, M. Headrick, and S. Minwalla, J. High Energy Phys. 10, 068 (2002), hep-th/0209002.

[12] U. Gürsoy, Vector operators in the BMN correspondence, hep-th/0208041; Predictions for PP-wave string amplitudes from perturbative SYM, hep-th/0212118.

[13] M. Spradlin and A. Volovich, Phys. Rev. D66, 086004 (2002), hep-th/0204146.

[14] Y. Kiem, Y. Kim, S. Lee, and J. Park, Nucl. Phys. B642, 389 (2002), hep-th/0205279.

[15] M.-x. Huang, Phys. Lett. B542, 255 (2002), hep-th/0205311.

[16] P. Lee, S. Moriyama, and J. Park, Phys. Rev. D66, 085021 (2002), hep-th/0206065.

[17] M. Spradlin and A. Volovich, J. High Energy Phys. 01, 036 (2003), hep-th/0206073

[18] C.-S. Chu, V. V. Khoze, M. Petrini, R. Russo, and A. Tanzini, A note on string interaction on the pp-wave background, hep-th/0208148. 
[19] A. Pankiewicz, J. High Energy Phys. 09, 056 (2002), hep-th/0208209.

[20] A. Pankiewicz and B. Stefański, Jr, Nucl. Phys. B657, 79 (2003), hep-th/0210246.

[21] Y. Kiem, Y. Kim, J. Park, and C. Ryou, J. High Energy Phys. 01, 026 (2003), hep-th/0211217.

[22] M. Billo' and I. Pesando, Phys. Lett. B536, 121 (2002), hep-th/0203028.

[23] A. Dabholkar and S. Parvizi, Nucl. Phys. B641, 223 (2002), hep-th/0203231.

[24] K. Skenderis and M. Taylor, J. High Energy Phys. 06, 025 (2002), hep-th/0205054.

[25] O. Bergman, M. R. Gaberdiel, and M. B. Green, D-brane interactions in type IIB planewave background, hep-th/0205183.

[26] P. Bain, K. Peeters, and M. Zamaklar, Phys. Rev. D67, 066001 (2003), hep-th/0208038.

[27] Y. Hikida and S. Yamaguchi, J. High Energy Phys. 01, 072 (2003), hep-th/0210262.

[28] K. Skenderis and M. Taylor, Open strings in the plane wave background I: Quantization and symmetries, hep-th/0211011.

[29] K. Skenderis and M. Taylor, Open strings in the plane wave background II: Superalgebras and Spectra, hep-th/0212184.

[30] M. R. Gaberdiel and M. B. Green, The $D$-instantons and other supersymmetric $D$-branes in IIB plane-wave string theory, hep-th/0211122.

[31] M. B. Green and J. H. Schwarz, Nucl. Phys. B218, 48 (1983).

[32] M. B. Green, J. H. Schwarz, and L. Brink, Nucl. Phys. B219, 437 (1983).

[33] M. B. Green and J. H. Schwarz, Nucl. Phys. B243, 475 (1984).

[34] R. R. Metsaev, Nucl. Phys. B655, 3 (2003), hep-th/0211178.

[35] B.-H. Lee, C. Park, and H. S. Yang, Open String Field Theory in Plane Wave Geometry, work in progress.

[36] N. D. Lambert and P. C. West, Phys. Lett. B459, 515 (1999), hep-th/9905031.

[37] J. Polchinski, String Theory: Superstring Theory and Beyond, (Cambridge Univ. Press, Cambridge, 1998), Vol. II, Chap. 10.

[38] O. DeWolfe, D. Z. Freedman, and H. Ooguri, Phys. Rev. D66, 025009 (2002), hep-th/0111135. 
[39] P. Lee and J. Park, Phys. Rev. D67, 026002 (2003), hep-th/0203257.

[40] K.-S. Cha, B.-H. Lee, and H. S. Yang, Supersymmetric p-p' Strings in Type IIB Plane Wave Background, work in progress. 